\title{
Multi-scale geometric analysis of Lagrangian structures in isotropic turbulence
}

\author{
YUE YANG广ं, D. I. PULLIN AND IVÁN BERMEJO-MORENO \\ Graduate Aerospace Laboratories, 205-45, California Institute of Technology, Pasadena, CA 91125, USA
}

(Received 20 July 2009; revised 27 January 2010; accepted 28 January 2010;

first published online 17 May 2010)

We report the multi-scale geometric analysis of Lagrangian structures in forced isotropic turbulence and also with a frozen turbulent field. A particle backwardtracking method, which is stable and topology preserving, was applied to obtain the Lagrangian scalar field $\phi$ governed by the pure advection equation in the Eulerian form $\partial_{t} \phi+\boldsymbol{u} \cdot \nabla \phi=0$. The temporal evolution of Lagrangian structures was first obtained by extracting iso-surfaces of $\phi$ with resolution $1024^{3}$ at different times, from $t=0$ to $t=T_{e}$, where $T_{e}$ is the eddy turnover time. The surface area growth rate of the Lagrangian structure was quantified and the formation of stretched and rolled-up structures was observed in straining regions and stretched vortex tubes, respectively. The multi-scale geometric analysis of Bermejo-Moreno \& Pullin (J. Fluid Mech., vol. 603, 2008, p. 101) has been applied to the evolution of $\phi$ to extract structures at different length scales and to characterize their non-local geometry in a space of reduced geometrical parameters. In this multi-scale sense, we observe, for the evolving turbulent velocity field, an evolutionary breakdown of initially large-scale Lagrangian structures that first distort and then either themselves are broken down or stretched laterally into sheets. Moreover, after a finite time, this progression appears to be insensible to the form of the initially smooth Lagrangian field. In comparison with the statistical geometry of instantaneous passive scalar and enstrophy fields in turbulence obtained by Bermejo-Moreno \& Pullin (2008) and Bermejo-Moreno et al. (J. Fluid Mech., vol. 620, 2009, p. 121), Lagrangian structures tend to exhibit more prevalent sheet-like shapes at intermediate and small scales. For the frozen flow, the Lagrangian field appears to be attracted onto a stream-surface field and it develops less complex multi-scale geometry than found for the turbulent velocity field. In the latter case, there appears to be a tendency for the Lagrangian field to move towards a vortex-surface field of the evolving turbulent flow but this is mitigated by cumulative viscous effects.

\section{Introduction}

In the paradigm of the energy cascade, three-dimensional turbulence is often viewed as composed of different scales with energy transferred from large scales to small scales in a self-similar process. A hierarchy of vortex sizes appears to be involved in this multi-stage process. In recent work, the multi-scale geometrical decomposition of instantaneous passive scalar (Bermejo-Moreno \& Pullin 2008), enstrophy and dissipation fields (Bermejo-Moreno, Pullin \& Horiuti 2009) showed a geometrical 
progression from blobs through tubes to sheet-like structures with decreasing physical length scales. Because the Eulerian fields analysed are obtained at a particular time instant, their geometrical decomposition does not unfold or clarify the geometry of the dynamical eddy evolution or breakdown process itself. This is an important question that is pertinent to cascade ideas put forward by Richardson that have been cast in similarity and statistical form by Kolmogorov and others. Furthermore, the knowledge of geometry of turbulent structures can inform a vorticity-based, smallscale description of turbulence (Lundgren 1982; Pullin \& Saffman 1993) from which subgrid-scale models suitable for large-eddy simulation (LES) can be constructed (e.g. Misra \& Pullin 1997; Chung \& Pullin 2009). To investigate the temporal evolution of turbulent structures, Lagrangian methods can be useful. In particular, for inviscid flow, vortex surfaces can be considered as Lagrangian structures (material surfaces). Relevant problems are the mechanism of transition from laminar flow to turbulent flow (e.g. Brachet et al. 1983) or the possible finite-time singularity in Euler dynamics (e.g. Boratav \& Pelz 1994; Hou \& Li 2006). An improved knowledge of Lagrangian structures can also help elucidate various applications in fluid dynamics, for example, scalar mixing (Warhaft 2000), premixed combustion (Pope 1987) and aquatic animal locomotion (Peng \& Dabiri 2008).

The main purpose of this work is to describe the non-local, multi-scale geometry of Lagrangian structures in the cascade process in turbulence. In Fourier space, the velocity field can be projected onto Fourier basis functions that represent, in some statistical sense, the hierarchy of eddy sizes. Although it is natural to present the energy spectrum in Fourier space, it is often difficult to attribute physical meaning to the amplitudes of the basis-function coefficients in terms, for example, of structural elements such as vortical structures with different geometry like tubes and sheets. An attractive physically intuitive idea of energy cascade might be cast in terms of vortex dynamics where vortex stretching is a crucial agent. But the participating eddies may not be the often portrayed cartoon, blob-like structures of different sizes. While sheet and tubes are attractive alternative geometries, there exist few relevant quantitative models with predictive or even postdictive capability. When the Reynolds number is infinite, vortex lines and surfaces can be considered as material lines and surfaces that are progressively stretched by chaotic motion in the inertial range to form highly convoluted shapes. This mechanism can occur at all scales, but the most efficient transfer of energy is caused by the interaction of vortices with similar sizes. There is, however, no explicit length scale in the vorticity equation in physical space. This suggests that a multi-scale method based on transforms with basis functions that are localized both in Fourier space and physical space is required (e.g. Meneveau 1991; Farge 1992).

The Lagrangian method for the study of turbulence, originated by Taylor (1922), is a classical but challenging approach, which involves tracking the trajectories of fluid particles. Recent progress has seen the combination of modern supercomputation and advanced experimental facilities, thus offering real promise for advancing our understanding of Lagrangian turbulence (see Toschi \& Bodenschatz 2009). To date, most studies of Lagrangian turbulence have focused on the statistics of single particles (see Yeung 2002), particle pairs (see Sawford 2001) and particle triangles or tetrahedrons (e.g. Pumir, Shraiman \& Chertkov 2000). Earlier, Batchelor (1952b) showed that the area of material surface elements increases exponentially as a consequence of conservation of mass of the fluid. The Lagrangian-history, direct interaction approximation developed by Kraichnan (1965) can provide quantitative predictions of single particle or particle-pair statistics in isotropic turbulence. Signature 
stretching and folding effects on Lagrangian structures in low-Reynolds-number flows were demonstrated in the chaotic advection theory of Aref (1984). Several stochastic models for local statistical and geometric structure in three-dimensional isotropic turbulence based on the properties of the velocity gradient tensor have been developed to show the material deformation history of fluid elements (e.g. Girimaji \& Pope 1990; Chertkov, Pumir \& Shraiman 1999; Li \& Meneveau 2007). To date, however, no general theory for Lagrangian turbulence exists, especially one that clarifies the non-local geometry (in the surface sense) of finite-sized Lagrangian structures in turbulence.

At the level of diagnostics of large numerical databases obtained from both experiment and direct numerical simulation (DNS), several non-local methodologies have recently been developed for the purpose of identifying structures in turbulence. A scheme for defining 'Lagrangian coherent structures' in three-dimensional flows was constructed by Haller (2001) using direct Lyapunov exponents along fluid trajectories. An extended structural and fractal description of turbulence has been proposed by Moisy \& Jiménez (2004) utilizing a box-counting method applied to sets of points of intense vorticity and strain-rate magnitude. Extended dissipation elements were defined by Wang \& Peters (2006) as the ensemble of grid cells from which the same pair of extremal points of the scalar field can be reached. While recent progress in particle-tracking techniques in turbulent experiments has demonstrated a promising capability for investigation of two-particle dispersion and Lagrangian tetrahedrons in three-dimensional turbulence (e.g. Bourgoin et al. 2006; Xu, Ouellette \& Bodenschatz 2008; Salazar \& Collins 2009), in order to follow coherent Lagrangian structures, tens and hundreds of thousands or even millions of particles need to be tracked simultaneously and instantaneously under some specified topological order. This task seems formidable for current experimental facilities. Finally, for numerical simulation, tracking finite-sized Lagrangian structure requires huge computational resources. In evolution, the geometry of Lagrangian structures typically becomes highly convoluted with some portions almost singular and hard to resolve (Pope, Yueng \& Girimaji 1989; Goto \& Kida 2007; Leonard 2009). Constraints on structure evolution are that the topology should be invariant and the volume conserved. Hence, a stable and topology-preserving method is required to track Lagrangian structures.

A topic closely related to the Lagrangian description is scalar advection-diffusion at very high Schmidt numbers in turbulence where mixing of passive tracers occurs with an extremely small molecular diffusivity. Recent work has confirmed the existence of intermittently distributed sheet-like structures for scalar gradients (Ruetsch \& Maxey 1992; Brethouwer, Hunt \& Nieuwstadt 2003) or scalar variance dissipation (Schumacher, Sreenivasan \& Yeung 2005). Some iso-contour plots can mimic geometry properties in Lagrangian turbulence but not in a rigorous sense, because there is no smallest scale in Lagrangian scalar dispersion without diffusion and the topology of iso-surfaces of the Lagrangian scalar must be invariant in evolution.

In this study, we address the multi-scale geometric analysis of Lagrangian structures in isotropic turbulence through DNS. In $\S 2$, a systematic framework is introduced to describe Lagrangian structures by the Lagrangian scalar field in turbulence. In $\S 3$, we will describe a backward particle-based method for tracking Lagrangian structures. In $\S 4$, on the basis of numerical results and theoretical estimations, we then consider the area growth rate of Lagrangian surfaces and discuss the formation of stretched and rolled-up structures with local flow patterns. Section 5 describes our application of the multi-scale geometric analysis developed by Bermejo-Moreno \& Pullin (2008) 
to investigate the non-local geometry of Lagrangian structures in time evolution at different length scales. Some conclusions are drawn in $\S 6$.

\section{Description of Lagrangian structures in turbulence}

\subsection{Lagrangian dynamic equations for incompressible flow}

Because the Lagrangian description is related directly to motion of individual fluid particles, it can provide a different perspective to the Eulerian description for the study of turbulent transport or the deformation of material surfaces and lines in a turbulent flow (see Monin \& Yaglom 1975). In this section, we will present a brief literature survey on the description of Lagrangian structures in turbulence and establish a formal theoretical framework in this study.

The trajectory of a fluid particle can be calculated by solving the kinematic equation

$$
\frac{\partial \boldsymbol{X}}{\partial t}=\boldsymbol{V}
$$

where $\boldsymbol{X}=\boldsymbol{X}\left(\boldsymbol{X}_{0}, t_{0} \mid t\right)$ is the location at time $t$ of the fluid particle that was located at $\boldsymbol{X}_{0}$ at the initial time $t_{0}$ with $\boldsymbol{X}=\left(X_{1}, X_{2}, X_{3}\right)$ and $\boldsymbol{X}_{0}=\left(X_{01}, X_{02}, X_{03}\right)$, and $\boldsymbol{V}=\boldsymbol{V}\left(\boldsymbol{X}_{0}, t_{0} \mid t\right)$ is the velocity at time $t$ of the fluid particle with $\boldsymbol{V}=\left(V_{1}, V_{2}, V_{3}\right)$. We use an upper case letter to denote a Lagrangian variable and a lower case letter for an Eulerian variable. The Lagrangian dynamic equation of incompressible flow is (see Monin \& Yaglom 1975)

$$
\begin{aligned}
\frac{\partial \boldsymbol{V}}{\partial t}=\frac{1}{\rho}\left[X_{j}, X_{k}, p\right]+v\left\{\left[X_{2}, X_{3},\left[X_{2}, X_{3}, \frac{\partial X_{i}}{\partial t}\right]\right]\right. & \\
+ & {\left.\left[X_{3}, X_{1},\left[X_{3}, X_{1}, \frac{\partial X_{i}}{\partial t}\right]\right]+\left[X_{1}, X_{2},\left[X_{1}, X_{2}, \frac{\partial X_{i}}{\partial t}\right]\right]\right\}, }
\end{aligned}
$$

where $\rho$ is the constant fluid density, $p$ is the pressure, $v$ is the kinematic viscosity, and the abbreviated notation for the Jacobians is

$$
[A, B, C]=\frac{\partial(A, B, C)}{\partial\left(X_{01}, X_{02}, X_{03}\right)} \text {. }
$$

The numerical solution of either (2.1) and (2.2) or the equations of the equivalent continuum-mechanics formulation (e.g. Marsden \& Hughes 1994) is formidable owing to the cubic and fifth-order nonlinearity for the pressure term and the viscous term respectively in the right-hand side of (2.2). Alternatively, the Lagrangian velocity for a fluid particle can be expressed as its local Eulerian velocity

$$
\boldsymbol{V}\left(\boldsymbol{X}_{0}, t_{0} \mid t\right)=\boldsymbol{u}\left(\boldsymbol{X}\left(\boldsymbol{X}_{0}, t_{0} \mid t\right), t\right),
$$

which can be solved for individual particles using a prior solution of the NavierStokes equation in Eulerian coordinates.

\subsection{Lagrangian infinitesimal line and surface elements}

The evolution of Lagrangian infinitesimal line elements $\boldsymbol{l}=\boldsymbol{X}^{(1)}-\boldsymbol{X}^{(2)}$ between a pair of fluid particles and (vector) surface elements $\boldsymbol{A}=\boldsymbol{l}^{(1)} \times \boldsymbol{l}^{(2)}$ in turbulence was first analysed by Batchelor (1952b). Because the volume of closed Lagrangian surfaces is conserved and any material line is stretched because of the convective nature of turbulence, the surface area $A(t)$ of Lagrangian structures will increase with time in evolution. Batchelor (1952b) proposed the exponential growth of the surface area $A$ 
for infinitesimal material elements

$$
A(t) \sim A_{0} \exp (\xi t),
$$

where $\xi$ is the growth rate and $A_{0} \equiv A(t=0)$, which was then verified numerically by Girimaji \& Pope (1990).

By tracking the Cauchy-Green tensor of deformation in isotropic turbulence, Girimaji \& Pope (1990) found that an initially spherical infinitesimal volume of fluid deforms into an ellipsoid with tube-like or sheet-like shapes in a finite time. Similar results were obtained by Pumir et al. (2000) using Lagrangian tetrahedrons. The evolution of infinitesimal elements and the Lagrangian models based on local velocity gradient tensors can provide valuable insight into the geometry of small-scale Lagrangian structures. These approaches cannot however, elucidate the geometry of finite-sized Lagrangian structures that could exhibit multiple scales with differing local geometries in evolution.

\subsection{Lagrangian scalar field and finite-sized Lagrangian structures}

Because two fluid particles, however close initially, tend to separate in turbulent flow (see Sawford 2001), a finite-sized Lagrangian structure cannot be described by the product of infinitesimal line elements for long times. Its motion can be expressed as an ensemble of particles comprising a material surface where each particle is marked by a constant scalar value in evolution in the interval $t_{0}$ to $t$

$$
\phi\left(X\left(\boldsymbol{X}_{0}, t_{0} \mid t\right), t\right)=\phi\left(\boldsymbol{X}_{0}, t_{0}\right) .
$$

A scalar field can be associated with these particles

$$
\phi(x, t)=\int_{\mathbb{R}^{3}} \psi(\boldsymbol{x}, t) \phi\left(\boldsymbol{X}\left(\boldsymbol{X}_{0}, t_{0} \mid t\right), t\right) \mathrm{d} \boldsymbol{X},
$$

by the Lagrangian position function

$$
\psi(\boldsymbol{x}, t) \equiv \delta\left(\boldsymbol{x}-\boldsymbol{X}\left(\boldsymbol{X}_{0}, t_{0} \mid t\right)\right) .
$$

From (2.6) and (2.7), the initial scalar field at $t=t_{0}$ can be written as

$$
\phi\left(\boldsymbol{x}, t_{0}\right)=\int_{\mathbb{R}^{3}} \psi\left(\boldsymbol{x}, t_{0}\right) \phi\left(\boldsymbol{X}_{0}, t_{0}\right) \mathrm{d} \boldsymbol{X}_{0},
$$

where

$$
\psi\left(\boldsymbol{x}, t_{0}\right)=\delta\left(\boldsymbol{x}-\boldsymbol{X}_{0}\right) .
$$

The Lagrangian position function $\psi(x, t)$ is determined by the particle trajectory $\boldsymbol{X}\left(\boldsymbol{x}_{0}, t_{0} \mid t\right)$, solving (2.1) either forward or backward in time. Thus, from (2.7)-(2.10) an instantaneous scalar field can be mapped to the initial scalar field as

$$
\phi(x, t) \longleftrightarrow \phi_{0} \equiv \phi\left(\boldsymbol{x}, t_{0}\right),
$$

by an ensemble of marked particles at $t_{0}$ and $t$ and their trajectories or Lagrangian characteristics represented by Lagrangian position functions $\psi(\boldsymbol{x}, t)$.

In fact the motion of marked particles can be related to a kind of scalar diffusion (Batchelor 1952a). Differentiating (2.8) with respect to time yields the equation of motion for $\psi$

where

$$
\frac{\partial \psi}{\partial t}=-\frac{\partial X_{i}}{\partial t} \delta_{i}^{\prime}(\boldsymbol{x}-\boldsymbol{X}),
$$

$$
\delta_{1}^{\prime}(\boldsymbol{x}-\boldsymbol{X})=\delta^{\prime}\left(x_{1}-X_{1}\right) \delta\left(x_{2}-X_{2}\right) \delta\left(x_{3}-X_{3}\right),
$$


with

$$
\delta^{\prime}\left(x_{1}-X_{1}\right)=\frac{\partial}{\partial x_{1}} \delta\left(x_{1}-X_{1}\right) .
$$

Substituting (2.1) and (2.4) into (2.12), we obtain

$$
\frac{\mathrm{D} \psi}{\mathrm{D} t}=\frac{\partial \psi}{\partial t}+u_{i} \frac{\partial \psi}{\partial x_{i}}=0 .
$$

Next, integrating (2.13) with an ensemble of marked particles $\phi\left(\boldsymbol{X}_{0}, t_{0}\right)$ at any initial time $t_{0}$ as

$$
\int_{\mathbb{R}^{3}} \frac{\mathrm{D} \psi}{\mathrm{D} t} \phi\left(\boldsymbol{X}_{0}, t_{0}\right) \mathrm{d} \boldsymbol{X}_{0}=\frac{\mathrm{D}}{\mathrm{D} t} \int_{\mathbb{R}^{3}} \psi\left(\boldsymbol{x}, t_{0}\right) \phi\left(\boldsymbol{X}_{0}, t_{0}\right) \mathrm{d} \boldsymbol{X}_{0}=0
$$

together with the mapping (2.11) of $\phi(x, t)$ between two time instants shows that the marked particle problem is a special case of the passive scalar dispersion without molecular diffusion

$$
\frac{\partial \phi}{\partial t}+\boldsymbol{u} \cdot \nabla \phi=0
$$

In this paper, we will use the term 'Lagrangian scalar' to denote the non-diffusive passive scalar whose evolution is described by (2.15). The function $\phi(x, t)$ will be described as a Lagrangian scalar field. The multi-scale geometric analysis of Lagrangian structures will later be obtained from the statistical geometry of isosurfaces of $\phi(\boldsymbol{x}, t)$ at different scales.

\subsection{Spectrum of the Lagrangian scalar field}

Under the straining motion of a velocity field $\boldsymbol{u}(\boldsymbol{x}, t)$, Lagrangian material surfaces that are iso-surfaces of $\phi(x, t)$ governed by (2.15) are stretched and folded, which will generally amplify the local scalar gradient and thereby cause the characteristic length scale of the scalar field to continually decrease. To investigate this cascade process of the Lagrangian scalar field in a periodic domain, we first consider the Fourier expansions

$$
\begin{aligned}
& \boldsymbol{u}(\boldsymbol{x}, t)=\sum_{\boldsymbol{k}} \hat{\boldsymbol{u}}(\boldsymbol{k}, t) \mathrm{e}^{\mathrm{i} k \cdot \boldsymbol{x}}, \\
& \phi(\boldsymbol{x}, t)=\sum_{\boldsymbol{k}} \hat{\phi}(\boldsymbol{k}, t) \mathrm{e}^{\mathrm{i} \boldsymbol{k} \cdot \boldsymbol{x}} .
\end{aligned}
$$

Substituting into (2.15) then gives

$$
\frac{\partial}{\partial t} \hat{\phi}(\boldsymbol{k}, t)+\mathrm{i} k_{m} \sum_{\boldsymbol{k}=\boldsymbol{p}+\boldsymbol{q}} \hat{u}_{m}(\boldsymbol{p}, t) \hat{\phi}(\boldsymbol{q}, t)=0,
$$

which expresses the interaction of wavenumber triads $\boldsymbol{k}, \boldsymbol{p}$, and $\boldsymbol{q}$. The equation of the scalar spectrum density

$$
\Phi(\boldsymbol{k}, t)=\langle\hat{\phi}(\boldsymbol{k}, t) \hat{\phi}(-\boldsymbol{k}, t)\rangle
$$

can then be obtained from (2.18) by multiplying $\hat{\phi}(-\boldsymbol{k}, t)$ and averaging

$$
\frac{\partial}{\partial t} \Phi(\boldsymbol{k}, t)+\mathrm{i} k_{m} \sum_{\boldsymbol{k}=\boldsymbol{p}+\boldsymbol{q}}\left\langle\hat{u}_{m}(\boldsymbol{p}, t) \hat{\phi}(\boldsymbol{q}, t) \hat{\phi}(-\boldsymbol{k}, t)\right\rangle=0 .
$$

In general, the Lagrangian scalar cascade transports $\Phi(\boldsymbol{k}, t)$ to higher and higher wavenumbers by the nonlinear interaction of wavenumber triads $\boldsymbol{k}=\boldsymbol{p}+\boldsymbol{q}$ without 
dissipation. In high-Reynolds-number turbulence, as $t \rightarrow \infty$, highly random velocity Fourier modes in the inertial range appear to redistribute scalar Fourier modes into all the wavenumbers with similar $\Phi(\boldsymbol{k}, t)$. In isotropic turbulence, the scalar spectrum is

$$
E_{\phi}(k, t)=4 \pi k^{2} \Phi(k, t)
$$

where $k=|\boldsymbol{k}|$. Hence, the Lagrangian scalar spectrum in isotropic turbulence at high Reynolds numbers may be expected to approach the asymptotic scaling law, at least within some high wavenumber ranges

$$
E_{\phi}(k, t) \sim O\left(k^{2}\right), \quad t \rightarrow \infty,
$$

which implies that the Lagrangian scalar field with a smooth initial condition may become discontinuous or else develop exponentially small structures as $t \rightarrow \infty$. In other words, the Lagrangian scalar field with finite spatial resolution over long times appears to become a spatial delta-correlated field $\langle\phi(\boldsymbol{x}) \phi(\boldsymbol{x}+\boldsymbol{r})\rangle=\delta(\boldsymbol{r})$.

From (2.18), however, the Lagrangian scalar field is still able to develop exponentially small-scale structures even in a steady, low-Reynolds-number flow, which is referred as 'chaotic advection' (Aref 1984). The asymptotic scaling law of the scalar spectrum in this case may depend on the specific flow, because the fixed velocity wavenumber vector in (2.18) may drive the Lagrangian scalar cascade in a particular direction.

In the sequel, we distinguish between Lagrangian turbulence dynamics and kinematics. The Kolmogorov-Obukhov-Corrsin theory (Monin \& Yaglom 1975) states that, in high-Reynolds-number flow, the cascade process of a passive scalar is controlled by the large-eddy turnover time $T_{e}$ independent of molecular viscosity. This implies that for $t>T_{e}$, the cascade is dominated by motions with scales smaller than the Kolmogorov length scale, which may be of lesser importance for the Lagrangian dynamics of turbulence. We therefore choose $T_{e}$ as the largest time in the investigation of the time evolution of Lagrangian structures. Although the analysis above indicates that the characteristic scale of Lagrangian structures decreases as time increases, Fourier-space representation is not well suited to an investigation of the finite geometry of the Lagrangian scalar field and corresponding non-local Lagrangian structures. This issue will be addressed in $\S 4.2$ by analysis of the scalar-gradient alignment and in $\S 5$ by multi-scale geometric decomposition.

\section{Simulation overview}

\subsection{Direct numerical simulation}

The Navier-Stokes equations for forced homogeneous and isotropic turbulence in a periodic box of side $L=2 \pi$ are written in the general form as

$$
\left.\begin{array}{l}
\frac{\partial \boldsymbol{u}}{\partial t}=\boldsymbol{u} \times \boldsymbol{\omega}-\nabla\left(\frac{p}{\rho}+\frac{1}{2}|\boldsymbol{u}|^{2}\right)+v \nabla^{2} \boldsymbol{u}+\boldsymbol{f}(\boldsymbol{x}, t), \\
\nabla \cdot \boldsymbol{u}=0,
\end{array}\right\}
$$

where $\omega \equiv \nabla \times \boldsymbol{u}$ is the vorticity. In this study, the flow was driven by a random forcing $\boldsymbol{f}(\boldsymbol{x}, t)$, which is non-zero for the Fourier modes with the wavenumber magnitude less than two.

The DNS of isotropic and homogeneous turbulence was performed using a standard pseudo-spectral method on a $256^{3}$ grid. The flow domain was discretized uniformly into $N^{3}$ grid points. Aliasing errors were removed using the two-thirds truncation 
Total kinetic energy

Mean dissipation rate

Root-mean-square velocity fluctuation

Taylor micro length scale

Taylor-Reynolds number

Kolmogorov length scale

Kolmogorov time scale

Spatial resolution

Integral length scale

Eddy turnover time

$\begin{array}{lc}E_{\text {tot }}=\left\langle\sum_{k} \hat{\boldsymbol{u}} \hat{\boldsymbol{u}}^{*}\right\rangle / 2 & 0.915 \\ \epsilon=2 v\left\langle\sum_{\boldsymbol{k}} k^{2} \hat{\boldsymbol{u}} \hat{\boldsymbol{u}}^{*}\right\rangle & 0.141 \\ u^{\prime}=\left(2 E_{\text {tot }} / 3\right)^{1 / 2} & 0.781 \\ \lambda_{T}=\left(15 v u^{\prime 2} / \epsilon\right)^{1 / 2} & 0.804 \\ \operatorname{Re}_{\lambda}=u^{\prime} \lambda_{T} / v & 62 \\ \eta=\left(v^{3} / \epsilon\right)^{1 / 4} & 0.052 \\ \tau_{\eta}=(v / \epsilon)^{1 / 2} & 0.266 \\ k_{\max } \eta & 4.3 \\ L_{e}=\left(\pi / 2 u^{\prime 2}\right) \int \mathrm{d} k E(k) / k & 1.76 \\ T_{e}=L_{e} / u^{\prime} & 2.26\end{array}$

TABLE 1. Summary of DNS parameters.

method. A stationary turbulence was generated by maintaining constant total energy in each of the first two wavenumber shells, with the energy ratio between the two shells consistent with $k^{-5 / 3}$. The spatial resolution in spectral simulation is evaluated as $k_{\max } \eta$, where $\eta \equiv(v / \epsilon)^{1 / 4}$ is the Kolmogorov length scale and the maximum wavenumber $k_{\max }$ is about $N / 3$. Proper resolution of the Kolmogorov scale requires $k_{\max } \eta>1$. The value of $k_{\max } \eta$ was typically larger than 4.3 in our simulation to ensure that we obtained accurate velocity fields for further Lagrangian computations. The Fourier coefficients of the flow velocity were advanced in time using a second-order Adams-Bashforth method. The time step was chosen to ensure that the CourantFriedrichs-Lewy (CFL) number was less that 0.5 for numerical stability and accuracy.

Table 1 lists parameters of the DNS flow fields used in this study, and figure 1 plots the resulting energy spectrum. Corresponding Lagrangian statistics for the same boxisotropic turbulent flow at similar Reynolds numbers in DNS and LES are reported in Yang, He \& Wang (2008). To demarcate Lagrangian dynamics and Lagrangian kinematics discussed in $\$ 2.4$, we will investigate the time evolution of Lagrangian structures in two separate cases. The first, referred to as a 'turbulent velocity field', consists of $\boldsymbol{u}(\boldsymbol{x}, t)$ obtained from the previously described DNS in $0 \leqslant t \leqslant T_{e}$. The second, described as a 'frozen velocity field' consists of an instantaneous field from the DNS $\boldsymbol{u}(\boldsymbol{x}, t=0)$. The frozen field was the result of running the DNS for $t<0$ of the order of several $T_{e}$. It is therefore an instantaneous snapshot from a fully developed stationary turbulent field but remains frozen in time for the purposes of solving (2.15). The instantaneous energy spectrum of $\boldsymbol{u}(\boldsymbol{x}, t=0)$ is quantitatively similar to figure 1 .

\subsection{Backward particle-tracking method}

Equation (2.15) is equivalent to the diffusion-less limit of the advection-diffusion equation in Eulerian form. The Eulerian finite-difference method for this pure hyperbolic equation exhibits significant numerical dissipation when the scalar gradient is high (e.g. LeVeque 1992), which is common in the evolution of Lagrangian scalar fields. To avoid this, we convert the Eulerian equation (2.15) to a set of ordinary differential equations as (2.1) to compute trajectories of fluid particles, which is equivalent to tracing characteristics of (2.15). Another potential problem is that if particles are tracked forward in time from $t_{0}$, they will be distributed almost randomly in space at later times, making it hard to reconstruct a continuous scalar field with satisfactory resolution on a Cartesian grid.

Instead, to obtain the Lagrangian field at a particular time $t$, we applied a backward particle-tracking method, which is absolutely stable, to deal with the convection term 


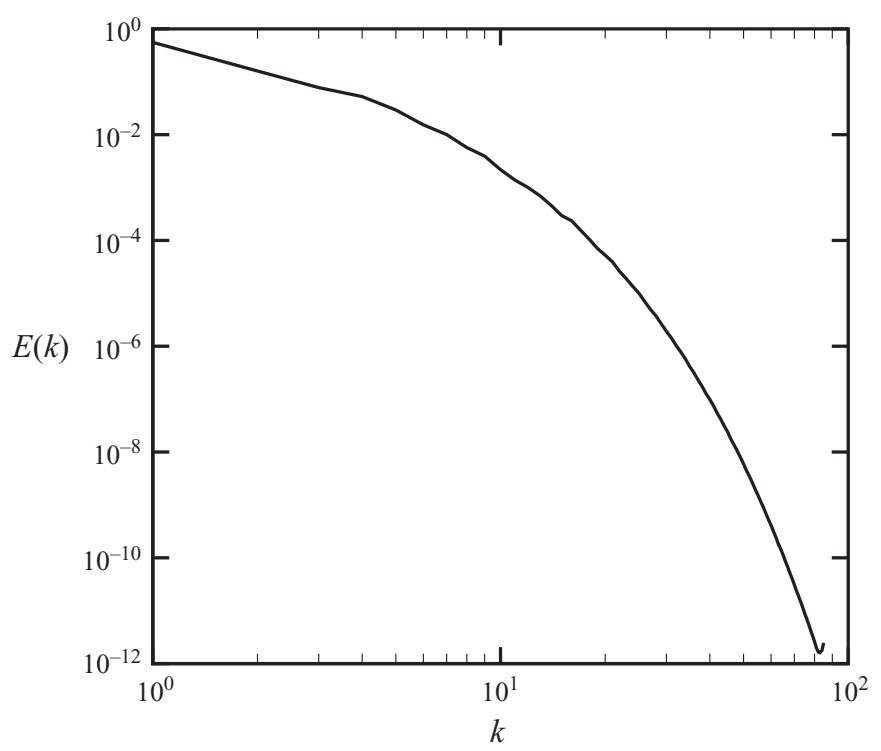

FIGURE 1. Energy spectrum of turbulence.

(e.g. Stam 1999; Nahum \& Seifert 2006). At time $t$, particles are placed at the grid points of $N_{p}^{3}$, where $N_{p}$ could be greater than the grid number $N$ of velocity field, i.e. the resolution of the Lagrangian scalar field could be higher than the velocity field. In this study, $N_{p}=1024$, which is four times the resolution of the velocity field. Then, particles are released and their trajectories calculated by solving (2.1). A three-dimensional fourth-order Lagrangian interpolation scheme is used to calculate fluid velocity at the location of a particle. The trajectory of a particle is then obtained by the explicit second-order Adams-Bashforth scheme. The time increment is selected to capture the finest resolved scales in the velocity field. All the accuracy of numerical schemes and parameters in this study for particle tracking satisfies the criteria proposed by Yeung \& Pope (1988) so that the computation of particle trajectories or Lagrangian characteristics are sufficiently accurate.

It is noted that the Eulerian velocity field here is reversed in time. In the simulation, we save the Eulerian velocity fields from DNS on disk at every time step and subsequently perform backward tracking from the particular time $t$ to the initial time $t_{0}$ with the reversed Eulerian velocity fields saved previously. After the backward tracking, we can obtain initial locations of particles $\boldsymbol{X}_{0}$, which is equivalent to obtaining the Lagrangian position function $\psi(\boldsymbol{x}, t)$ for each particle located at the grid at $t$. From a given initial condition consisting of a smooth Lagrangian field $\phi\left(\boldsymbol{x}, t_{0}\right)$, we can obtain the Lagrangian field at time $t$ on the Cartesian grid by (2.6) and (2.7), or the simple mapping (2.11) with Lagrangian coordinates and position functions.

In implementation, we store the position $\boldsymbol{X}_{0}$ at $t=0$ and $\boldsymbol{X}\left(\boldsymbol{X}_{0}, t_{0} \mid t\right)$ at a given time for each particle with the same index as binary files after the backward tracking, which is equivalent to saving the information of $\psi(\boldsymbol{x}, t)$ of all the particles. Then we can apply arbitrarily many different initial conditions to obtain corresponding Lagrangian scalar fields at the particular $t$ from the same particle position files $\boldsymbol{X}_{0}$ and $\boldsymbol{X}\left(\boldsymbol{X}_{0}, t_{0} \mid t\right)$ in a single run. The tradeoff is that independent simulations are required for each time $t$ at which $\phi(x, t)$ is sought. Because $\phi(x, t)$ is obtained via the direct mapping (2.11) from the initial field, the probability density function (p.d.f.) of 
$\phi(\boldsymbol{x}, t)$ is invariant with time and the scalar fluctuation variance

$$
\operatorname{Var}(\phi) \equiv\left\langle(\phi(\boldsymbol{x}, t)-\langle\phi(\boldsymbol{x}, t)\rangle)^{2}\right\rangle
$$

is also invariant with time. Hence, in principle there is no numerical dissipation in the computation of the Lagrangian scalar field by the backward particle-tracking method.

As $t \rightarrow \infty$, the Lagrangian scalar field with a smooth initial condition may become discontinuous or else develop exponentially small structures. If the backward particle integration from $t$ to $t_{0}$ was exact for each particle, then the solution values on the $N_{p}^{3}$ grid would correspond to

$$
\begin{aligned}
\phi(i \Delta x, j \Delta y, k \Delta z, t) & =\int_{0}^{L} \int_{0}^{L} \int_{0}^{L} \phi(x, y, z, t) \delta(x-i \Delta x) \delta(y-j \Delta y) \delta(z-k \Delta z) \mathrm{d} x \mathrm{~d} y \mathrm{~d} z \\
i & =1, \ldots, N_{p}, \quad j=1, \ldots, N_{p}, \quad k=1, \ldots, N_{p}
\end{aligned}
$$

where $\phi(x, y, z, t)$ on the right-hand side is the exact continuous solution. Thus, our calculation of $\phi$ at time $t$ on the discrete field would be exact but interpolated values may have $O(1)$ errors for sufficiently large $t$. In practice, we find that the largest time for which we can obtain accurate, smooth and well-resolved Lagrangian scalar fields simulated at the present resolution $1024^{3}$ is the large-eddy turnover time $T_{e}$ defined in table 1.

To illustrate the Lagrangian scalar field at longer times using higher resolution with the backward particle-tracking method, we place particles at one cross-section (or any desired sub-domain) of the entire three-dimensional field prior to tracking. We can then obtain the Lagrangian scalar field in a plane cut. Figure 2(a) shows the Lagrangian scalar field on a plane cut $0 \leqslant x \leqslant 2 \pi, 0 \leqslant y \leqslant 2 \pi, z=\pi$ with a $4096^{2}$ grid at $t=2.5 T_{e}$ from a smooth initial condition $\phi_{0}=\cos x$ at $t=t_{0}=0$ driven by a turbulent velocity field. The scalar field has become extremely convoluted with signature stretched and spiral structures. The effects of resolution for a detail of the scalar field, in the sense of (3.2), are shown in figure $2(b-d)$. This demonstrates the effectiveness of the backward particle-tracking method in capturing the finest scale features with increasing particle numbers for large $t$. The scalar field with the same initial condition and the same time lapse in the frozen velocity field is shown in figure 3. This shows substantially less detailed structure than for the turbulent flow. Because straining and vortex motions in the frozen field are fixed, the stretched and spiral structures are concentrated only in a small number of local regions.

\subsection{Three initial conditions of the Lagrangian scalar field}

In this study, we investigated the evolution of Lagrangian scalar fields with three different initial conditions $\phi_{0}^{(i)}$ in the same domain of the velocity field. The isosurfaces of these initial fields are closed surfaces. These are as follows:

(i) $\phi_{0}^{(1)}=g\left(x_{0}\right) g\left(y_{0}\right) g\left(z_{0}\right)$, where the Gaussian function

$$
g(x)=\exp \left(-0.5(x-\pi)^{2}\right) .
$$

The iso-surfaces of $\phi_{0}^{(1)}$ are concentric spheres/blob-like surfaces.

(ii) $\phi_{0}^{(2)}=g\left(x_{0}\right) g\left(y_{0}\right) F_{0}$, where the filter function $F_{0}=f\left(x_{0}\right) f\left(y_{0}\right) f\left(z_{0}\right)$ with

$$
f(x)=\exp \left(-6\left(\frac{x-\pi}{\pi}\right)^{6}\right),
$$


(a) Plane cut of the full 3D scalar field with resolution $4096^{2}, 0 \leq x \leq 2 \pi$ and $0 \leq y \leq 2 \pi$.

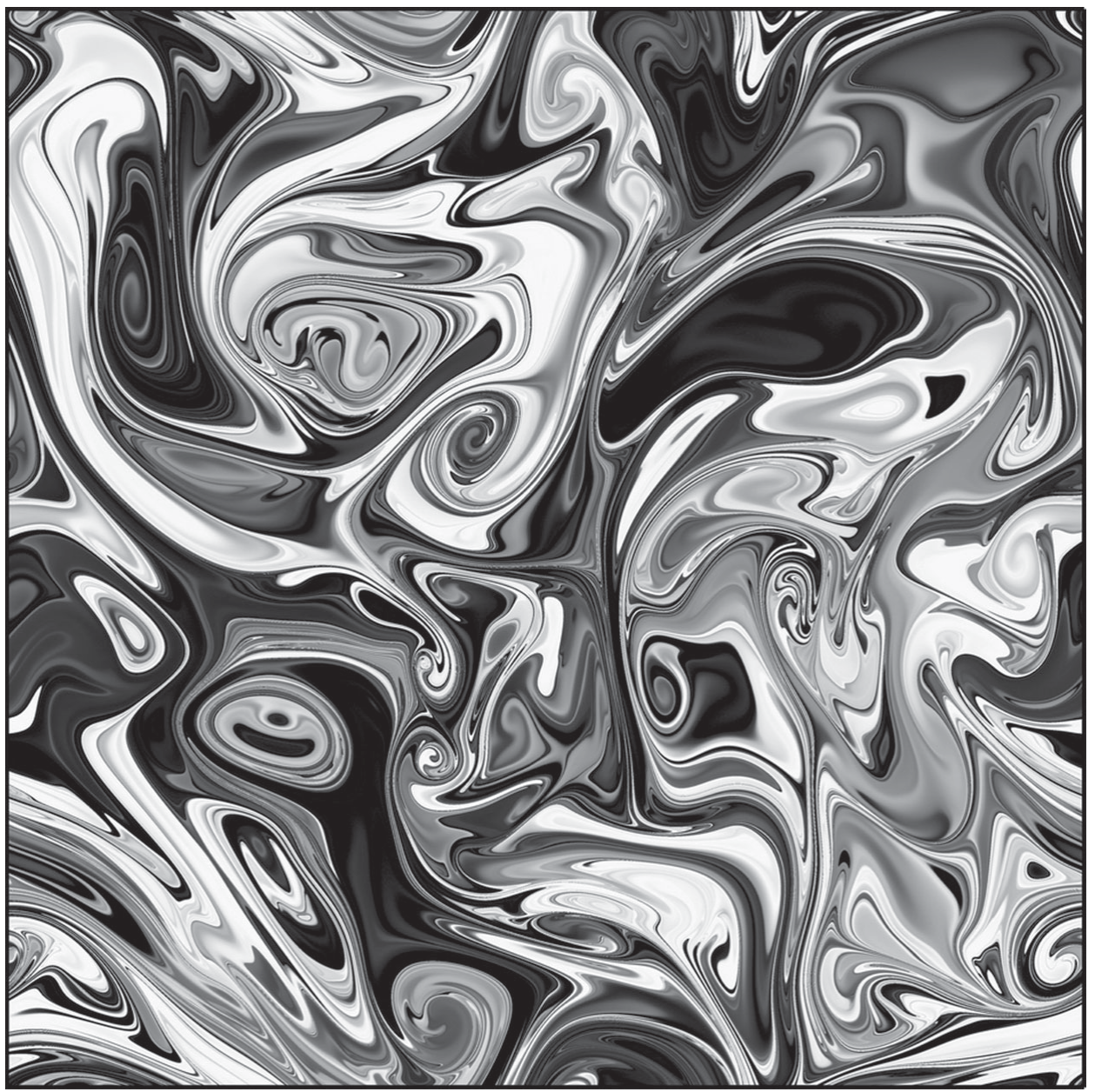

(b) $1024^{2}$

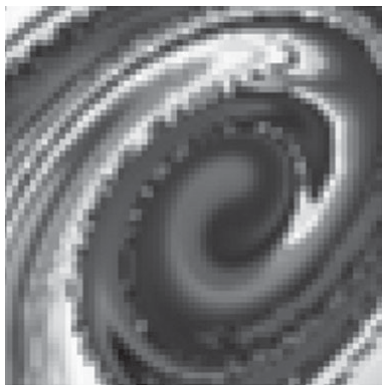

(c) $2048^{2}$

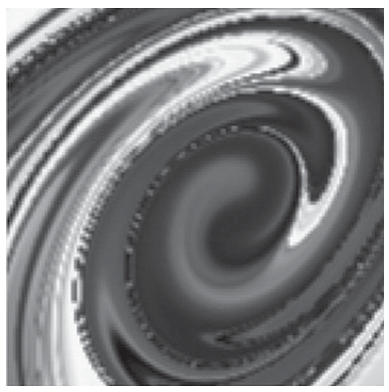

(d) $4096^{2}$

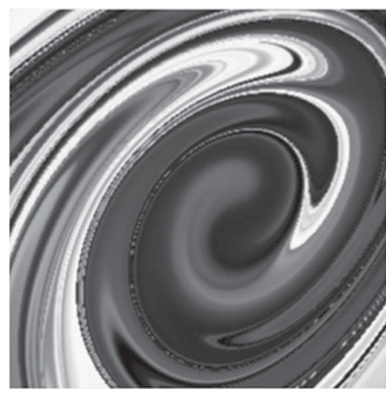

FiguRE 2. The $x-y$ plane cut at $z=\pi$ from the three-dimensional Lagrangian scalar field at $t=2.5 T_{e}$ with the initial condition $\phi_{0}=\cos x$ in the forced stationary homogeneous and isotropic turbulence, and zoomed parts of plane cuts at a small region $2.5 \leqslant x \leqslant 2.9$ and $3.6 \leqslant y \leqslant 4$ with increasing resolutions. 


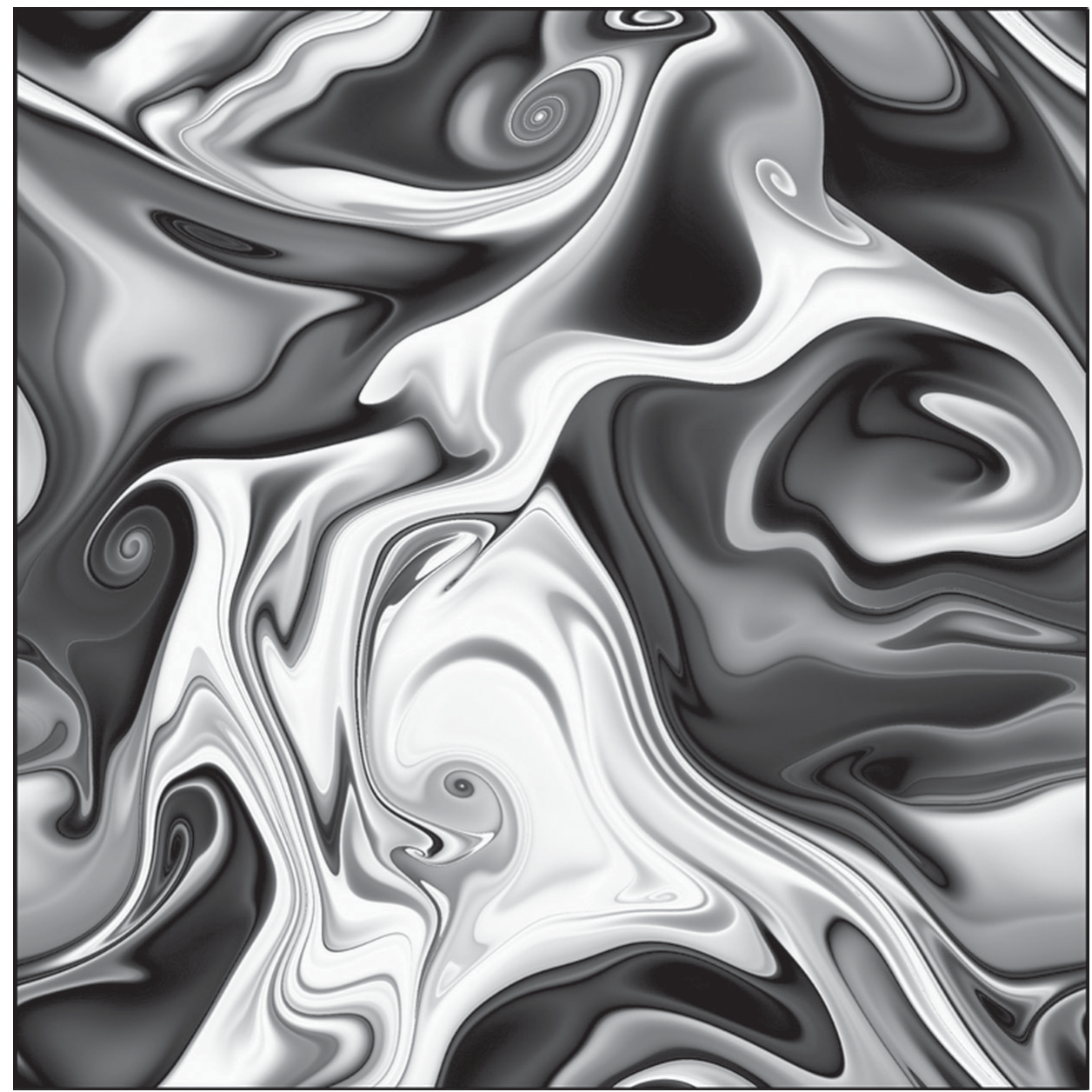

FIGURE 3. The $x-y$ plane cut at $z=\pi$ from the three-dimensional Lagrangian scalar field at $t=2.5 T_{e}$ with the initial condition $\phi_{0}=\cos x$ and resolution $4096^{2}$ in the frozen turbulent field.

which corresponds to iso-surfaces that are coaxial and tube-like in the $z$-direction. The filter function $F_{0}$ used here makes the corners of iso-surfaces curved for improved identification (Bermejo-Moreno \& Pullin 2008).

(iii) $\phi_{0}^{(3)}=g\left(x_{0}\right) F_{0}$, which corresponds to mostly parallel planes along the $x$-axis. Two typical plane cuts of these three initial fields are shown in figure 4 . The characteristic length scale of the initial fields is comparable with the integral length scale $L_{e}$ in turbulence. It is noted that topological properties of Lagrangian structures are invariant in time. For example, if an iso-surface is topological sphere (i.e. simply connected) at $t=0$, then it remains so for all finite $t$.

\section{Evolution of Lagrangian scalar fields}

\subsection{Growth rate of the surface area of Lagrangian structures}

The particle simulations for the Lagrangian scalar fields were done with $1024^{3}$ and correspond to an evolution time from initial conditions $\phi_{0}^{(i)}, i=1,2,3$, from $t=0$ to $t=T_{e}$. At times $t=\alpha T_{e}$, where $\alpha=\{1 / 16,1 / 8,1 / 4,1 / 2,3 / 4,1\}$, Lagrangian scalar 
(a) $g\left(x_{0}\right) g\left(y_{0}\right)$

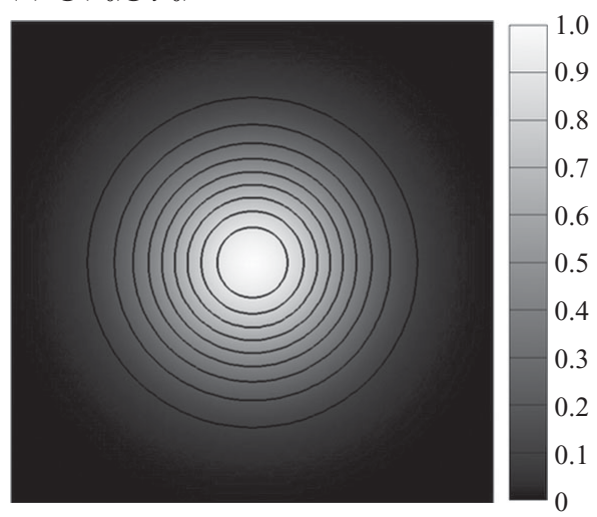

(b) $g\left(x_{0}\right) f\left(y_{0}\right)$

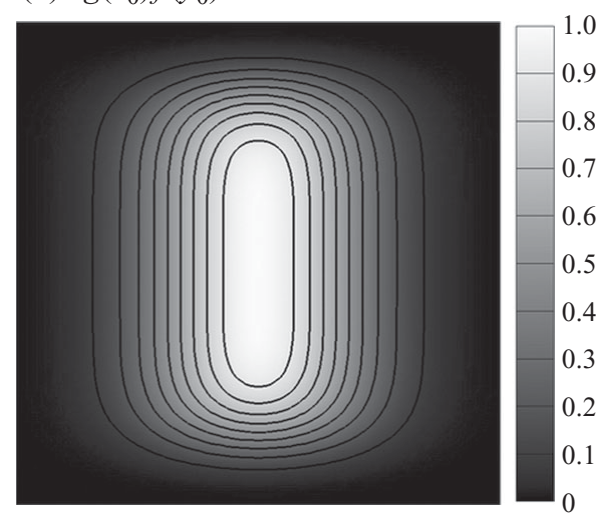

FIgURE 4. Typical plane cuts with iso-contour lines of three initial Lagrangian scalar fields.

(a) $t=0$

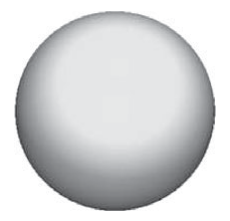

(d) $t=T_{e} / 4$

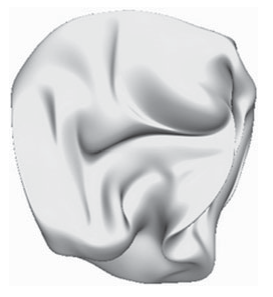

(b) $t=T_{e} / 16$

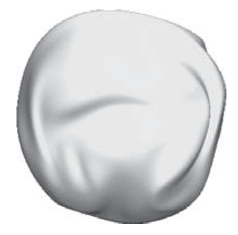

(e) $t=T_{e} / 2$

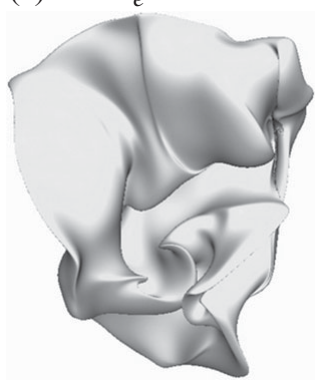

(c) $t=T_{e} / 8$

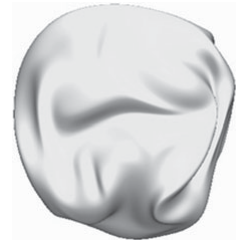

(f) $t=3 T_{e} / 4$

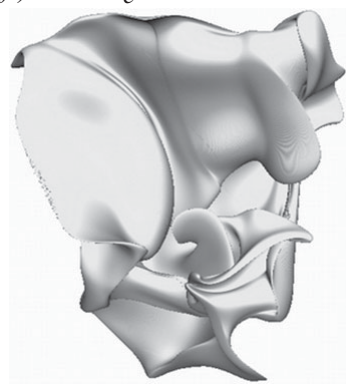

FIGURE 5. Iso-surfaces $(\phi=0.3)$ of three-dimensional Lagrangian scalar fields with the blob-like initial $\phi_{0}^{(1)}$ at different times.

fields $\phi\left(x, \alpha T_{e}\right)$ were obtained with three different initial conditions. For example, the evolution of the Lagrangian structure with the blob-like initial condition $\phi_{0}^{(1)}$ corresponding to the iso-surface $\phi=0.3$ in turbulence is shown in figure 5. From a simple sphere, after a finite time, the iso-surface is distorted to a highly convoluted shape with small-scale rolled-up structures. The spreading or widening of the spectrum of $\phi(t)$ in figure 6 shows a cascade process from large scales to small scales for the $\phi(t)$ field in stationary isotropic turbulence and the frozen turbulent field. The difference between spectra from two cases is very small at the early time, but then becomes noticeable with increasing time. The Lagrangian scalar cascade in turbulence is faster than that in the frozen field by dynamic convection motions. At a longer time, the difference in spectra should be more apparent as implied in figures 2 and 3. 


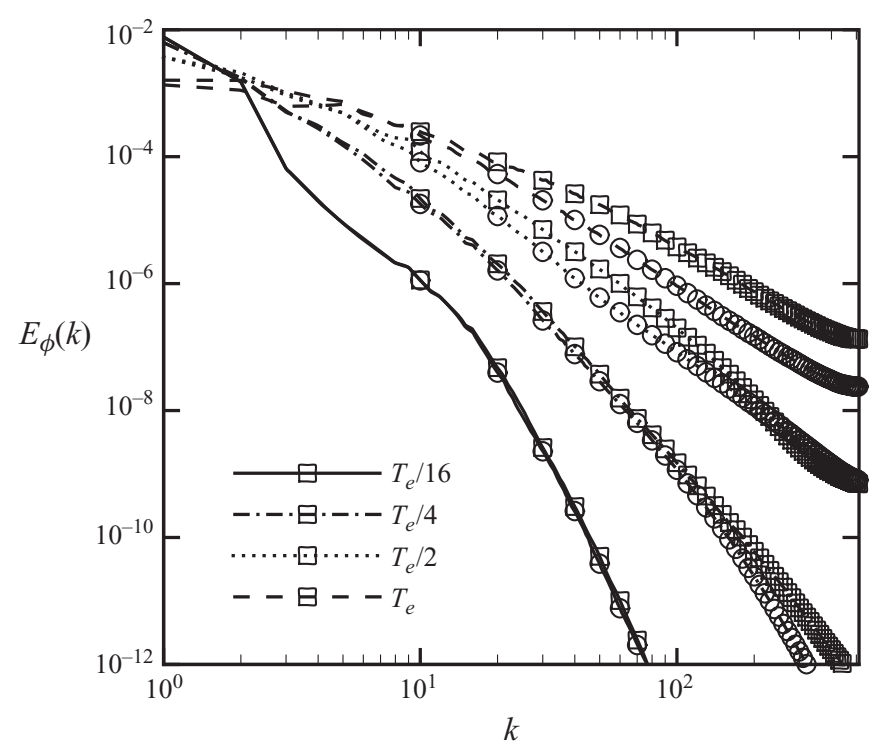

FIGURE 6. Spectra of Lagrangian scalar fields with the blob-like initial $\phi_{0}^{(1)}$ at different times in stationary isotropic turbulence $(\square)$ and the frozen turbulent field $(\bigcirc)$.

The exponential growth of the surface area $A(t)$ of Lagrangian structures, initially proposed by Batchelor (1952b) for infinitesimal material elements, was also verified numerically by Goto \& Kida (2007) using finite-sized material surfaces. To validate this in the present study, typical Lagrangian structures were selected as iso-surfaces at iso-contour levels $\phi=\{0.1,0.2, \ldots, 0.9\}$. Each iso-surface is discretized on a triangular mesh, and the total area is the sum of areas of all the individual triangles. The area growth rate

$$
\xi(t)=\frac{1}{t} \log \left(\frac{A(t)}{A_{0}}\right)
$$

is computed by (2.5), and then the average growth rate $\langle\xi(t)\rangle$ at a particular time was presently obtained by taking an average over iso-surfaces of $\phi$ at nine contour levels. As shown in figure 7, following a rapid, monotonic growth in $2 \sim 3$ Kolmogorov times, the average stretching rate approaches a statistical stationary state around $\xi=(0.33 \pm 0.4) \tau_{\eta}^{-1}$ in turbulence. This observation is consistent with that of Goto \& Kida (2007).

\subsection{Alignment of the Lagrangian scalar gradient in turbulent and frozen flows}

The evolutionary geometry of Lagrangian structures may be related to the turbulent velocity field or vorticity field by preferred alignments of the scalar gradient with the background vector fields. Batchelor (1952b) predicted that the vorticity $\omega$ and the Lagrangian scalar gradient $\nabla \phi$ tend to become perpendicular. To investigate the time evolution of this alignment, we define the cosine of the angle between these vectors as

$$
\lambda_{\omega}=\frac{\omega \cdot \nabla \phi}{|\omega||\nabla \phi|} .
$$

Similarly, the cosine of the angle between $\boldsymbol{u}$ and $\nabla \phi$ can be defined as

$$
\lambda_{u}=\frac{\boldsymbol{u} \cdot \nabla \phi}{|\boldsymbol{u}||\nabla \phi|} .
$$




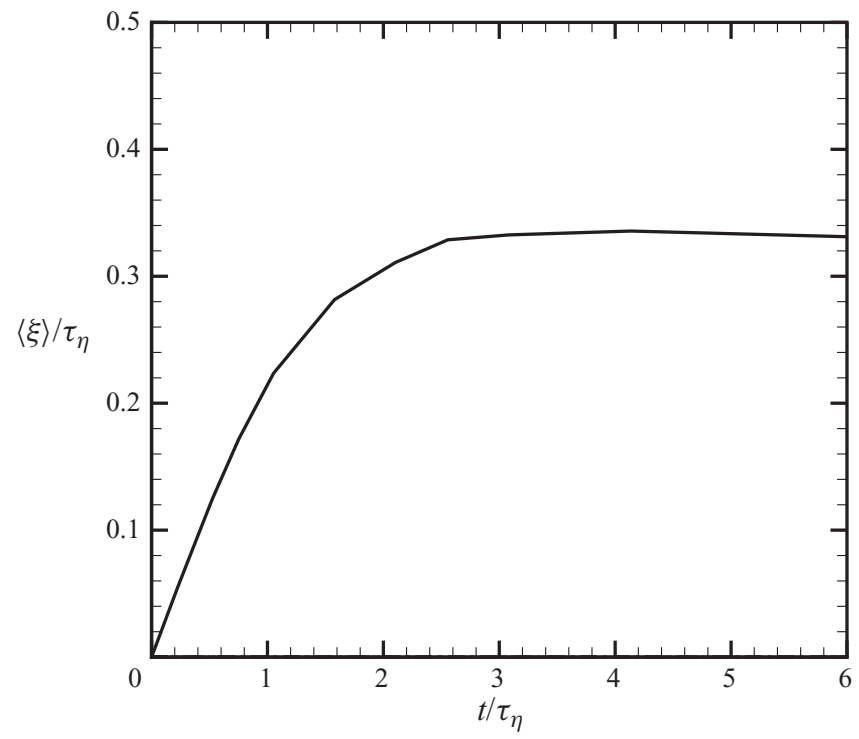

FIGURE 7. Temporal evolution of the average stretching rate $\langle\xi\rangle$ rescaled by the reciprocal of the Kolmogorov time scale $\tau_{\eta}$ of Lagrangian structures that are obtained from iso-surfaces of $\phi=\{0.1,0.2, \ldots, 0.9\}$ with $\phi_{0}^{(1)}$ in stationary isotropic turbulence.

Figure 8 shows the temporal evolution of p.d.f.s of $\left|\lambda_{\omega}\right|$ and $\left|\lambda_{u}\right|$ for both the turbulent (figure $8 a, b$ ) and the frozen (figure $8 c, d$ ) velocity fields. The p.d.f.s of $\left|\lambda_{\omega}\right|$ in isotropic turbulence in figure $8(a)$ indicates evolution from an almost uniform distribution in the initial condition at $t=0$ towards a strong peak at $\pi / 2$. We also find that $\nabla \phi$ tends to become normal to the local velocity as illustrated in figure $8(b)$ showing the p.d.f.s of $\left|\lambda_{u}\right|$. The time evolution of p.d.f.s of $\left|\lambda_{\omega}\right|$ and $\left|\lambda_{u}\right|$ in the frozen turbulent field is shown in figure $8(c, d)$. A comparison of figure $8(a)$ with figure $8(b)$ shows that Lagrangian surfaces in stationary turbulence tend to align with vortex lines rather than streamlines at $t=T_{e}$. In contrast, as shown in figures $8(c)$ and $8(d)$ Lagrangian surfaces advected by the frozen velocity field over long times appear to preferentially align with streamlines rather than vortex lines.

\subsection{Transport equations for alignment angles}

These observations on alignment angles can be understood by analysis of the transport equations for $\left|\lambda_{\omega}\right|$ and $\left|\lambda_{u}\right|$. We assume $\langle\boldsymbol{u}\rangle=0$. From (2.15), we can obtain the vector identity for $\nabla \phi, \boldsymbol{u}$, and a vector $\boldsymbol{v}$

$$
\frac{\mathrm{D}}{\mathrm{D} t}(\boldsymbol{v} \cdot \nabla \phi)=\left(\frac{\partial \boldsymbol{v}}{\partial t}+\boldsymbol{u} \cdot \nabla \boldsymbol{v}-\boldsymbol{v} \cdot \nabla \boldsymbol{u}\right) \cdot \nabla \phi,
$$

and then transport equations for $\boldsymbol{\omega} \cdot \nabla \phi$ and $\boldsymbol{u} \cdot \nabla \phi$ :

$$
\begin{gathered}
\frac{\mathrm{D}}{\mathrm{D} t}(\boldsymbol{\omega} \cdot \nabla \phi)=\left(\frac{\partial \boldsymbol{\omega}}{\partial t}+\nabla \times(\boldsymbol{\omega} \times \boldsymbol{u})\right) \cdot \nabla \phi, \\
\frac{\mathrm{D}}{\mathrm{D} t}(\boldsymbol{u} \cdot \nabla \phi)=\left(\frac{\partial \boldsymbol{u}}{\partial t}\right) \cdot \nabla \phi .
\end{gathered}
$$



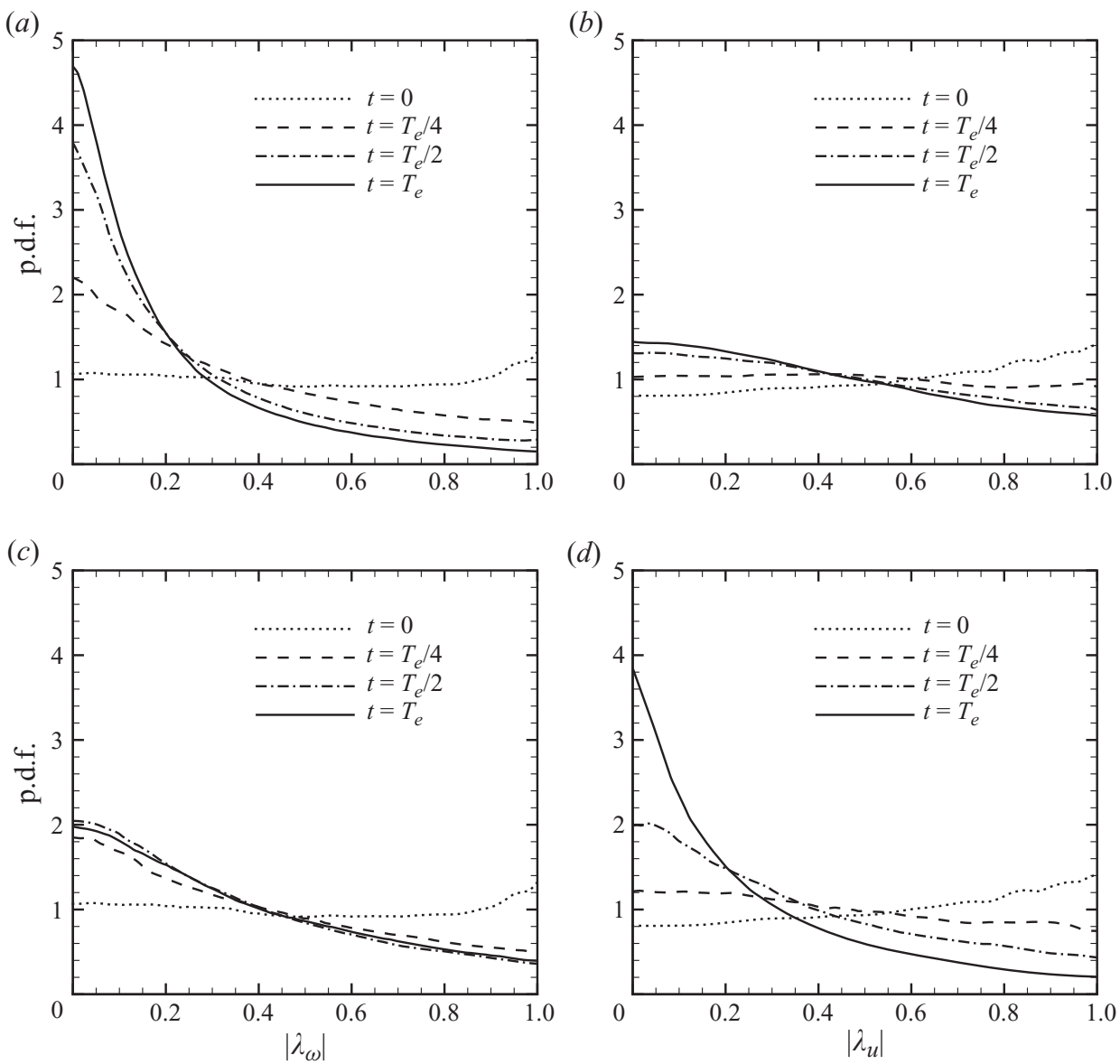

FIGURE 8. Temporal evolution of p.d.f.s of $\left|\lambda_{\omega}\right|$ and $\left|\lambda_{u}\right|$ obtained from the Lagrangian scalar field with $\phi_{0}^{(1)}$ in $(a, b)$ stationary isotropic turbulence and $(c, d)$ the frozen turbulent field, where $\lambda_{\omega}$ is the cosine of the angle between $\omega$ and $\nabla \phi$, and $\lambda_{u}$ is the cosine of the angle between $\boldsymbol{u}$ and $\nabla \phi$.

Introducing the unit vector $\boldsymbol{n}_{\phi}=\nabla \phi /|\nabla \phi|$, we can obtain the transport equation for $|\nabla \phi|$ from (2.15)

$$
\frac{\mathrm{D}|\nabla \phi|}{\mathrm{D} t}=-\left(\boldsymbol{n}_{\phi} \cdot \boldsymbol{s} \cdot \boldsymbol{n}_{\phi}\right)|\nabla \phi|,
$$

where $\boldsymbol{S}=\left(\partial u_{i} / \partial x_{j}+\partial u_{j} / \partial x_{i}\right) / 2$ is the rate-of-strain tensor. In an unsteady flow, from (4.5), (4.6) and the vorticity equation in a viscous flow

$$
\frac{\mathrm{D} \omega}{\mathrm{D} t}=\boldsymbol{\omega} \cdot \nabla \boldsymbol{u}+v \nabla^{2} \boldsymbol{\omega}
$$

we can derive equations for $\lambda_{\omega}$ and $\lambda_{u}$

$$
\begin{aligned}
& \frac{\mathrm{D} \lambda_{\omega}}{\mathrm{D} t}=\left(\boldsymbol{n}_{\phi} \cdot \boldsymbol{s} \cdot \boldsymbol{n}_{\phi}-\frac{1}{|\boldsymbol{\omega}|} \frac{\mathrm{D}|\boldsymbol{\omega}|}{\mathrm{D} t}\right) \lambda_{\omega}+\frac{v}{|\boldsymbol{\omega}|} \nabla^{2} \boldsymbol{\omega} \cdot \boldsymbol{n}_{\phi}, \\
& \frac{\mathrm{D} \lambda_{u}}{\mathrm{D} t}=\left(\boldsymbol{n}_{\phi} \cdot \boldsymbol{s} \cdot \boldsymbol{n}_{\phi}-\frac{1}{|\boldsymbol{u}|} \frac{\mathrm{D}|\boldsymbol{u}|}{\mathrm{D} t}\right) \lambda_{u}+\frac{1}{|\boldsymbol{u}|}\left(\frac{\partial \boldsymbol{u}}{\partial t}\right) \cdot \boldsymbol{n}_{\phi} .
\end{aligned}
$$


In the frozen velocity field, $\partial \boldsymbol{u} / \partial t=0$, and then

$$
\begin{gathered}
\frac{\mathrm{D}}{\mathrm{D} t}(\boldsymbol{\omega} \cdot \nabla \phi)=(\nabla \times(\boldsymbol{\omega} \times \boldsymbol{u})) \cdot \nabla \phi, \\
\frac{\mathrm{D}}{\mathrm{D} t}(\boldsymbol{u} \cdot \nabla \phi)=0,
\end{gathered}
$$

so the equations for $\lambda_{\omega}$ and $\lambda_{u}$ become

$$
\begin{gathered}
\frac{\mathrm{D} \lambda_{\omega}}{\mathrm{D} t}=\left(\boldsymbol{n}_{\phi} \cdot \boldsymbol{S} \cdot \boldsymbol{n}_{\phi}-\frac{1}{|\boldsymbol{\omega}|} \boldsymbol{u} \cdot \nabla|\boldsymbol{\omega}|\right) \lambda_{\omega}+\frac{1}{|\boldsymbol{\omega}|}(\nabla \times(\boldsymbol{\omega} \times \boldsymbol{u})) \cdot \boldsymbol{n}_{\phi}, \\
\frac{\mathrm{D} \lambda_{u}}{\mathrm{D} t}=\left(\boldsymbol{n}_{\phi} \cdot \boldsymbol{S} \cdot \boldsymbol{n}_{\phi}-\boldsymbol{n}_{u} \cdot \nabla|\boldsymbol{u}|\right) \lambda_{u},
\end{gathered}
$$

where $\boldsymbol{n}_{u}=\boldsymbol{u} /|\boldsymbol{u}|$.

On a fluid particle, the solution of (4.7) is given by

$$
|\nabla \phi(t)|=\left|\nabla \phi\left(t_{0}\right)\right| \exp \left(-\int_{t_{0}}^{t} \boldsymbol{n}_{\phi} \cdot \boldsymbol{s} \cdot \boldsymbol{n}_{\phi} \mathrm{d} t^{\prime}\right) .
$$

For both the turbulent and the frozen velocity fields we may write for the scalar gradient

$$
\langle|\nabla \phi(t)|\rangle=\left(2 \int_{0}^{\infty} k^{2} E_{\phi}(k, t) \mathrm{d} k\right)^{1 / 2} .
$$

A consequence of the widening scalar spectrum $E_{\phi}(k, t)$ discussed in $\S 2.4$ and (4.16), where the integral is weighted by $k^{2}$, is the progressive amplification of $\langle|\nabla \phi(t)|\rangle$. This implies that in (4.15), statistically

$$
\left\langle n_{\phi} \cdot \boldsymbol{S} \cdot \boldsymbol{n}_{\phi}\right\rangle<0
$$

Let the principal strain rates for $S$ be $\Gamma_{\alpha}, \Gamma_{\beta}, \Gamma_{\gamma}$, with corresponding unit vectors along the principal axes of strain $\boldsymbol{e}_{\alpha}, \boldsymbol{e}_{\beta}, \boldsymbol{e}_{\gamma}$. In incompressible flow, $\Gamma_{\alpha}+\Gamma_{\beta}+\Gamma_{\gamma}=0$ and we specify the order $\Gamma_{\alpha} \geqslant \Gamma_{\beta} \geqslant \Gamma_{\gamma}$. Ashurst et al. (1987) showed that statistically $\Gamma_{\alpha}>\Gamma_{\beta}>0$ and $\Gamma_{\gamma}<0$ in isotropic turbulence. In addition, the alignment vector $\lambda_{i}=\boldsymbol{n}_{\phi} \cdot \boldsymbol{e}_{i}$ is defined as the cosine of the angle between $\boldsymbol{n}_{\phi}$ and $\boldsymbol{e}_{i}$. Because the passive scalar gradient tends to align with the most compressive strain direction in turbulent flow (e.g. Ashurst et al. 1987; Ruetsch \& Maxey 1992; Brethouwer et al. 2003) and referring to (4.17), we hypothesize that in turbulent flow or the frozen turbulent flow,

$$
\left\langle\boldsymbol{n}_{\phi} \cdot \boldsymbol{s} \cdot \boldsymbol{n}_{\phi}\right\rangle=\left\langle\Gamma_{\alpha} \lambda_{\alpha}^{2}\right\rangle+\left\langle\Gamma_{\beta} \lambda_{\beta}^{2}\right\rangle+\left\langle\Gamma_{\gamma} \lambda_{\gamma}^{2}\right\rangle \sim O\left(\Gamma_{\gamma}\right)<0 .
$$

The magnitude of the term $\left\langle\boldsymbol{n}_{u} \cdot \nabla|\boldsymbol{u}|\right\rangle$ in (4.14) can be reasonably assumed small in comparison with that of $\left\langle\boldsymbol{n}_{\phi} \cdot \boldsymbol{S} \cdot \boldsymbol{n}_{\phi}\right\rangle$, because there is no preferred alignment between $\boldsymbol{n}_{u}$ and $\nabla|\boldsymbol{u}|$. Thus, from (4.14) and (4.18), in general the volume-averaged $\left|\lambda_{u}\right|$ can be expected to decrease exponentially with time in the frozen turbulent field as

$$
\left\langle\left|\lambda_{u}\right|\right\rangle \sim\left\langle\left|\lambda_{u 0}\right|\right\rangle \exp (\Gamma t)
$$

with $\Gamma<0$ and $\lambda_{u 0} \equiv \lambda_{u}(t=0)$. In other words, the $\phi$-field in the frozen velocity field is attracted to a stream-surface field whose iso-surfaces are stream surfaces comprised of steady stream lines.

On the other hand, $\mathrm{D}\langle|\omega|\rangle / \mathrm{D} t \approx 0$ for stationary turbulence, which implies that (4.9) could be simplified as

$$
\frac{\mathrm{D} \lambda_{\omega}}{\mathrm{D} t} \approx\left(\boldsymbol{n}_{\phi} \cdot \boldsymbol{s} \cdot \boldsymbol{n}_{\phi}\right) \lambda_{\omega}+v O\left(R_{\omega}\right),
$$




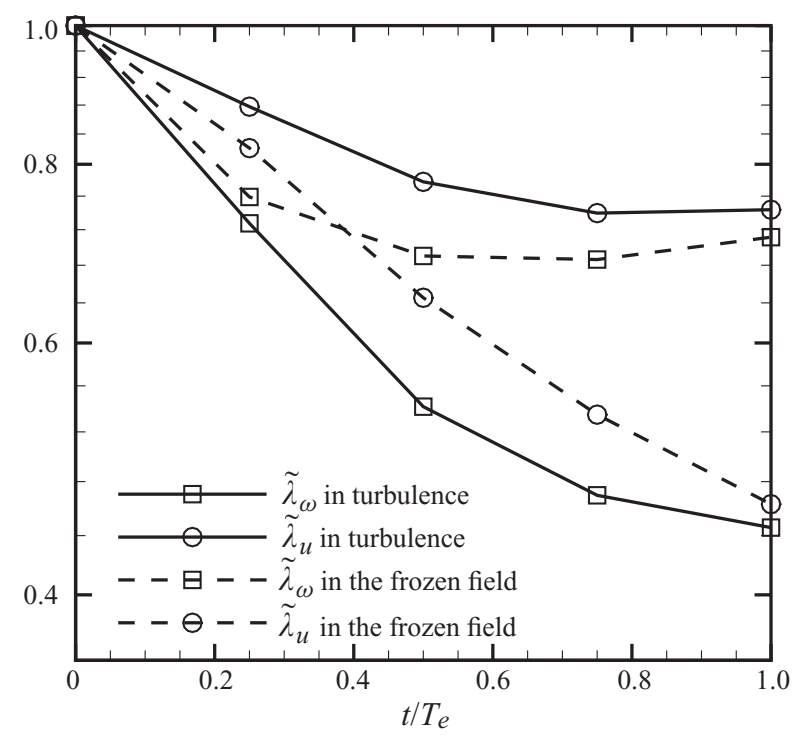

Figure 9. The log-linear plot of the temporal evolution of $\widetilde{\lambda}_{\omega}$ and $\widetilde{\lambda}_{u}$ in stationary isotropic turbulence and the frozen turbulent field.

where $v$ is small in high-Reynolds-number turbulent flow and $R_{\omega}=\left|\nabla^{2} \omega\right| /|\omega|$. By (4.20) the approximation for the volume-averaged $\left|\lambda_{\omega}\right|$ in turbulent flow could be

$$
\left\langle\left|\lambda_{\omega}\right|\right\rangle \sim\left\langle\left|\lambda_{\omega 0}\right|\right\rangle \exp (\Gamma t)+v \frac{O\left(R_{\omega}\right)}{\Gamma}(1-\exp (\Gamma t)),
$$

with $\Gamma<0$ and $\lambda_{\omega 0} \equiv \lambda_{\omega}(t=0)$. The exponential decay in (4.21) appears to be similar as that in (4.19) for small time $t$, which is then attenuated for larger $t$ owing to the cumulative effect of the small, inhomogeneous viscous term. Hence, the analysis suggests that $\phi$-field in stationary turbulence may tend initially to be attracted towards a vortexsurface field whose iso-surfaces are vortex surfaces comprised of vortex lines, but that perfect, long-time alignment is inhibited owing to small but persistent viscous effects.

Furthermore, the last inhomogeneous terms in both (4.10) and (4.13) could be non-trivial. Hence, the evolution of $\left\langle\left|\lambda_{u}\right|\right\rangle$ in turbulence may perhaps be described qualitatively by the model

$$
\left\langle\left|\lambda_{u}\right|\right\rangle \sim\left\langle\left|\lambda_{u 0}\right|\right\rangle \exp (\Gamma t)+\left\langle f_{u}\left(\lambda_{u}\right)\right\rangle,
$$

with a similar model for the evolution of $\left\langle\left|\lambda_{\omega}\right|\right\rangle$ in the frozen field

$$
\left\langle\left|\lambda_{\omega}\right|\right\rangle \sim\left\langle\left|\lambda_{\omega 0}\right|\right\rangle \exp (\Gamma t)+\left\langle f_{\omega}\left(\lambda_{\omega}\right)\right\rangle .
$$

Here, the functions $\left\langle f_{u}\left(\lambda_{u}\right)\right\rangle$ and $\left\langle f_{\omega}\left(\lambda_{\omega}\right)\right\rangle$ are not expected to be as small as the last term in (4.21) due to the additional non-trivial inhomogeneous terms in the governing equations of $\lambda_{\omega}$ and $\lambda_{u}$. This suggests that the Lagrangian scalar field appears to show only a weak tendency to be attracted to the stream-surface field in turbulence and to the vortex-surface field in the frozen field for long times.

The temporal evolution of the normalized volume-averaged statistics

$$
\tilde{\lambda}_{\omega} \equiv\left\langle\left|\lambda_{\omega}\right|\right\rangle /\left\langle\left|\lambda_{\omega 0}\right|\right\rangle \quad \text { and } \quad \tilde{\lambda}_{u} \equiv\left\langle\left|\lambda_{u}\right|\right\rangle /\left\langle\left|\lambda_{u}\right|\right\rangle
$$

is plotted in figure 9. As shown in (4.19), the evolution of $\widetilde{\lambda}_{u}$ in the frozen field shows strong exponential decay from $t=0$ to $t=T_{e}$, which implies that Lagrangian structures 
Cases

$\tilde{\lambda}_{\omega}$ in stationary isotropic turbulence

$\tilde{\lambda}_{u}$ in stationary isotropic turbulence

$\tilde{\lambda}_{\omega}$ in the frozen turbulent field

$\tilde{\lambda}_{u}$ in the frozen turbulent field
Approximation
Attractor

Vortex surface

Stream surface

Vortex surface

Stream surface
Tendency (early $\rightarrow$ late)

$$
\begin{aligned}
& \text { Strong } \rightarrow \text { weak } \\
& \text { Medium } \rightarrow \text { weak } \\
& \text { Medium } \rightarrow \text { weak } \\
& \text { Strong } \rightarrow \text { strong }
\end{aligned}
$$

TABLE 2. Summary of the evolution of alignments for the Lagrangian scalar field.

are progressively attracted to frozen stream surfaces. The evolution of $\tilde{\lambda}_{\omega}$ in isotropic turbulence shows even faster exponential decay from $t=0$ to $t=T_{e} / 2$ than that of $\widetilde{\lambda}_{u}$ in the frozen field, but then as estimated in (4.21), the viscous effect tends to slow down the decay corresponding to the tendency that Lagrangian structures become attracted to vortex surfaces in turbulence. Furthermore, the evolution of $\tilde{\lambda}_{u}$ in isotropic turbulence and $\widetilde{\lambda}_{\omega}$ in the frozen field rapidly approaches a statistical stationary state as shown in the approximations (4.22) and (4.23), which implies relatively weak alignments in both cases. These evolutions of alignments and corresponding tendencies of attractions of stream surfaces or vortex surfaces at early and late stages are summarized in table 2. Here, we remark that the numerical results for the alignment issue are based on the initial condition $\phi^{(1)}$ with the large initial length scale $L_{e}$. The corresponding results with initial conditions $\phi^{(2)}$ and $\phi^{(3)}$ show quantitatively similar behaviours.

\subsection{Formation of spiral and stretched Lagrangian structures}

An interesting observation from figure 5 is the rolled-up or spiral structures at later stages of the Lagrangian evolution. This phenomenon was discussed by Ruetsch \& Maxey (1992) as multiply layered sheets or spiral structures of strong passive scalar gradient when $S c \gg 1$. Previous studies (e.g. Ruetsch \& Maxey 1992; Brethouwer et al. 2003) observed that vortex tubes are surrounded by scalar gradient sheets over a wide range of Prandtl/Schimdt numbers. It is notable that rolled-up structures, perhaps generated by vortex tubes, have themselves been observed mainly in scalar simulations at high Schmidt numbers. These highly convoluted Lagrangian structures were denoted as 'folding effects' by Goto \& Kida (2007), who argued that the folding is produced by coherent counter-rotating eddy pairs. Presently, we hypothesize that the formation of spiral structures of $\phi$ in eddies corresponding to rolled-up shapes of Lagrangian structures can be also explained as the alignment discussed in $\$ \S 4.2$ and 4.3 between the gradient of $\phi$ and other vectors obtained from the local velocity field.

A simple kinematic model can be constructed: consider a two-dimensional exact solution of the scalar dispersion (e.g. Rhines \& Young 1983). The governing equations for scalar $\phi(r, \theta, t)$, vorticity $\omega(r, \theta, t)$ and streamfunction $\psi(r, \theta, t)$ in two-dimensional polar coordinates are

$$
\begin{gathered}
\frac{\partial \phi}{\partial t}+\frac{1}{r}\left(\frac{\partial \psi}{\partial \theta} \frac{\partial \phi}{\partial r}-\frac{\partial \phi}{\partial \theta} \frac{\partial \psi}{\partial r}\right)=0 \\
\frac{\partial \omega}{\partial t}+\frac{1}{r}\left(\frac{\partial \psi}{\partial \theta} \frac{\partial \omega}{\partial r}-\frac{\partial \omega}{\partial \theta} \frac{\partial \psi}{\partial r}\right)=0 \\
\omega=\left(\frac{1}{r} \frac{\partial}{\partial r} r \frac{\partial}{\partial r}+\frac{1}{r^{2}} \frac{\partial^{2}}{\partial \theta^{2}}\right) \psi
\end{gathered}
$$


(a) $t=0$

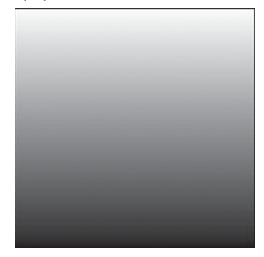

(b) $t=10$

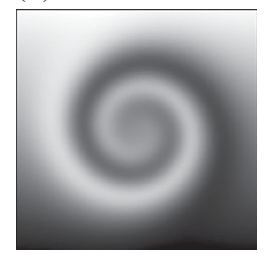

(c) $t=25$

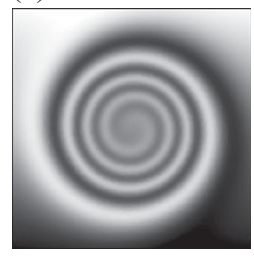

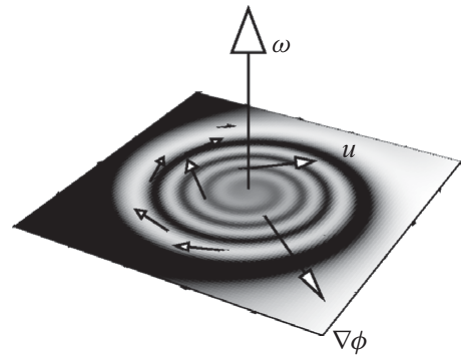

FIGURE 10. Evolution of spiral structures for the two-dimensional solution (4.28). The illustration of orthogonality between $\boldsymbol{u}$ and $\nabla \phi$ is shown in the rightmost subfigure.

For illustrative purposes we consider initial conditions corresponding to a linear scalar gradient embedded within an axisymmetric vortex

$$
\phi(r, \theta, 0)=r \sin \theta, \quad \omega(r, \theta, 0)=\frac{1}{r} \frac{\mathrm{d}}{\mathrm{d} r} r^{2} \Omega, \quad \Omega=\mathrm{e}^{-r^{2}},
$$

but emphasize that the following scenario is more general. The vortex remains steady while $\phi$ evolves as

$$
\phi(r, \theta, t)=r \sin (\theta-\Omega t) .
$$

The evolution of (4.28) is plotted in figure 10, where it is seen that the linear scalar gradient is wound into tight spirals by convective action of the vortex. It is straightforward to obtain $\lambda_{u}$ as

$$
\lambda_{u}=-\frac{\cos (\theta-\Omega t)}{\sqrt{\cos ^{2}(\theta-\Omega t)+\left[\sin (\theta-\Omega t)+2 \Omega r^{2} t \cos (\theta-\Omega t)\right]^{2}}} .
$$

At $t=0,\left|\lambda_{u}\right|=|\cos \theta|$, where the probability of $\theta$ is uniformly distributed in $0 \leqslant$ $\theta<2 \pi$, so the p.d.f. of $x=\left|\lambda_{u}\right|$ in the entire two-dimensional plane is a monotonic increasing function $(2 / \pi)\left(1-x^{2}\right)^{-1 / 2}$ with $0 \leqslant x<1$. As $t \rightarrow \infty$, from (4.29), $\left|\lambda_{u}\right| \rightarrow 0$ everywhere, so the p.d.f. of $\left|\lambda_{u}\right|$ is asymptotic to a delta function $\delta(x-\epsilon), \epsilon \rightarrow 0$. The evolution of the p.d.f. of $\left|\lambda_{u}\right|$ in this two-dimensional case is qualitatively consistent with that shown in figures $8(b)$ and $8(d)$. Since Lagrangian structures are topologically invariant, then starting from any $\phi_{0}$ except $\boldsymbol{u} \cdot \nabla \phi_{0}=0$, this model supports the hypothesis that the spiral is the most probable structure within eddies. In figure 11, we can visually observe spatial coincidences between spiral Lagrangian structures represented by iso-contour lines of $\phi\left(t=T_{e}\right)$ and vortical structures represented by regions of high enstrophy $\left(\omega_{i} \omega_{i}\right)^{1 / 2}$ in isotropic turbulence. Additionally, elongated Lagrangian structures induced by strong stretching motions are observed to wrap vortex tubes.

\subsection{Lagrangian $Q-R$ analysis}

The local topology of flow field can be classified by the invariants $Q$ and $R$ of the velocity gradient tensor $A_{i j}=\partial u_{i} / \partial x_{j}$ (Chong, Perry \& Cantwell 1990), where

$$
Q=-\frac{1}{2} A_{i j} A_{i j}, \quad R=-\frac{1}{2} A_{i j} A_{j k} A_{k i} .
$$

One way to quantify the relation between Lagrangian structures and local flow patterns in turbulence is to use the mean value of the norm of the scalar gradient $|\nabla \phi|$ conditioned on $Q$ and $R$ at $t=T_{e}$. We denote this by $\langle|\nabla \phi| \mid Q, R\rangle$. As shown in figure 12 , the curve $(27 / 4) R^{2}+Q^{3}=0$ divides the $R-Q$ plane into four regions, each 

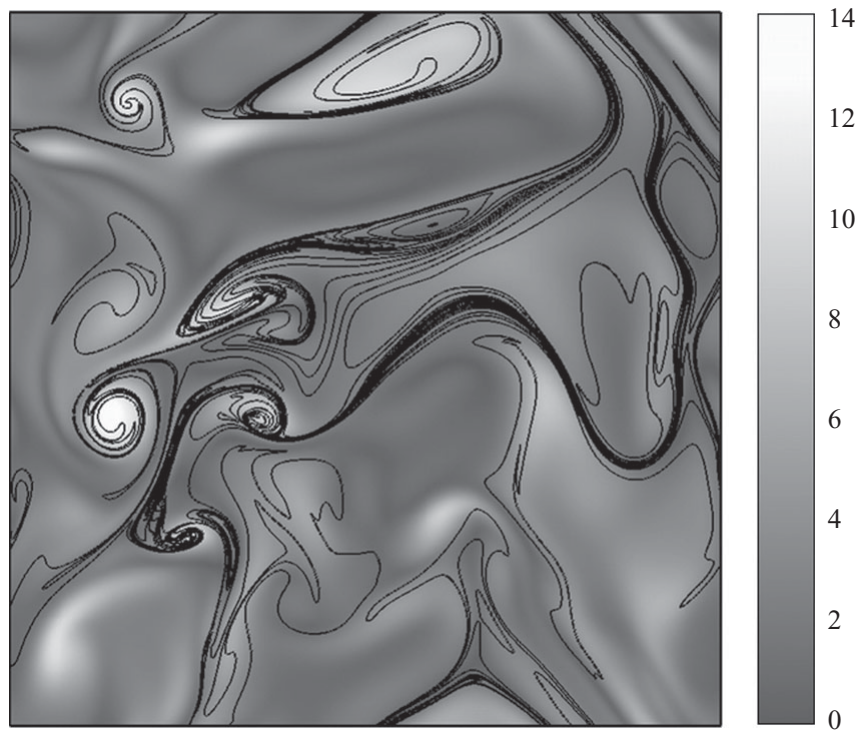

FIGURE 11. Iso-contour lines of $\phi\left(t=T_{e}\right)$ and contours of the enstrophy at $t=T_{e}$ on the $x-y$ plane cut at $z=\pi$ with the blob-like initial $\phi_{0}^{(1)}$ in stationary isotropic turbulence.

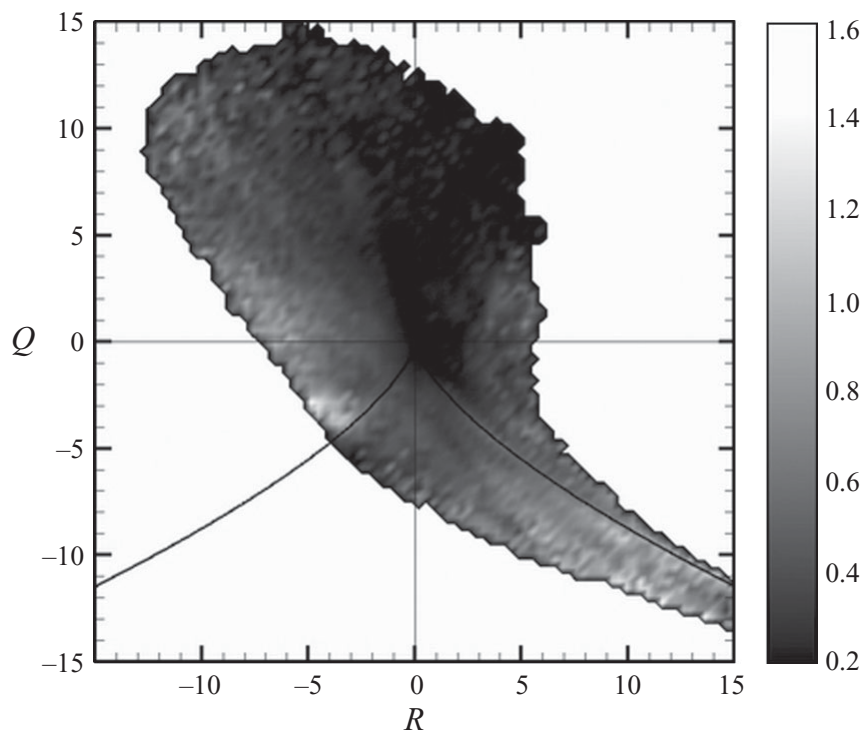

FIGURE 12. The mean value of the gradient $|\nabla \phi|$ of the Lagrangian scalar field conditioned on $Q$ and $R$ at $t=T_{e}$ in stationary isotropic turbulence. The whole plane is divided into four regions by the curve $(27 / 4) R^{2}+Q^{3}=0$. The local flow patterns for each region are upper left: stretched vortex; upper right: compressed vortex; lower left: axial strain; lower right: bi-axial strain.

with different local flow structure. Details of the local flow pattern for each region are described by Brethouwer et al. (2003) and references therein. Because the number of samples is too small outside the pear-shaped contour to determine an accurate $\langle|\nabla \phi| \mid Q, R\rangle$, the values in the white regions are ignored. We observe steep scalar gradients in regions dominated by bi-axial strains, which is consistent with the result 
obtained by scalar simulation with $S c=0.7$ from Brethouwer et al. (2003). This result corresponds to the scenario of stretched Lagrangian structures in straining regions. In particular, we also observe strong scalar gradients in regions inside stretched vortex tubes, which could correspond to the scenario of spiral Lagrangian structures in eddies as shown in figures 2 and 11.

\section{Multi-scale geometric analysis of Lagrangian structures}

\subsection{Description of the methodology}

In this section, we apply the multi-scale geometric methodology of Bermejo-Moreno \& Pullin (2008) to explore the non-local geometry of Lagrangian structures at different length scales. Starting from a given periodic three-dimensional field, this methodology has three main steps: extraction, characterization and classification. The extraction of structures is itself done in two main stages. In the first stage, the curvelet transform (see Candès et al. 2005) applied to the full three-dimensional Lagrangian scalar field at an instant in time provides a multi-scale decomposition into a finite set of component three-dimensional fields, one per scale. Second, by iso-contouring each component field at one or more iso-contour levels, a set of closed iso-surfaces is obtained that represent the structures at that scale.

The basis functions of the curvelet transform, which we refer to as 'curvelets', are oriented needle-shaped elements in space and a wedge in frequency, and they are localized in scale (Fourier space), position (physical space) and orientation. The projection of the scalar field onto the curvelet basis produces a set of decomposed fields, each characterized by a radial (in Fourier space) index $j$. Let the number of grid points in each spatial direction be $2^{n}$. Then for each scale $j=j_{0}, \ldots, j_{e}$, with $j_{0}=2, j_{e}=\log _{2}(n / 2)$, the associated curvelet radial basis function has support near the dyadic corona $\left[2^{j-1}, 2^{j+1}\right]$ in Fourier space. This gives a multi-scale decomposition of the original scalar field into a total of $j_{e}-j_{0}+1$ scale-dependent fields. For convenience we will subsequently label scale-dependent fields by the index $i=j-2$, $i=0, \ldots, j_{e}-2$, with $i=0$ corresponding to the largest-scale field and $i=j_{e}-2$ the smallest resolved-scale field. Curvelets are naturally suited for detecting, organizing or providing a compact representation of multi-dimensional structures. The latest studies on applications of curvelets to turbulence were reported by Bermejo-Moreno et al. (2009) and Ma et al. (2009).

The characterization stage begins with the joint p.d.f., in terms of area coverage on each individual surface, of two differential-geometry properties, the absolute value of the shape index $S$ and the dimensionless curvedness $C$,

$$
S \equiv\left|-\frac{2}{\pi} \arctan \left(\frac{\kappa_{1}+\kappa_{2}}{\kappa_{1}-\kappa_{2}}\right)\right|, \quad C \equiv 3 \frac{V}{A} \sqrt{\frac{\kappa_{1}^{2}+\kappa_{2}^{2}}{2}},
$$

where $\kappa_{1}$ and $\kappa_{2}$ are principal curvatures, $A$ is the surface area and $V$ is the volume contained within the three-dimensional surface. Characterization is based on the construction of a finite set of parameters defined by algebraic functions of dimensionless forms of first and second moments of the joint p.d.f. of $S$ and $C$ for each individual surface. An additional parameter,

$$
\lambda \equiv \sqrt[3]{36 \pi} \frac{V^{2 / 3}}{A},
$$



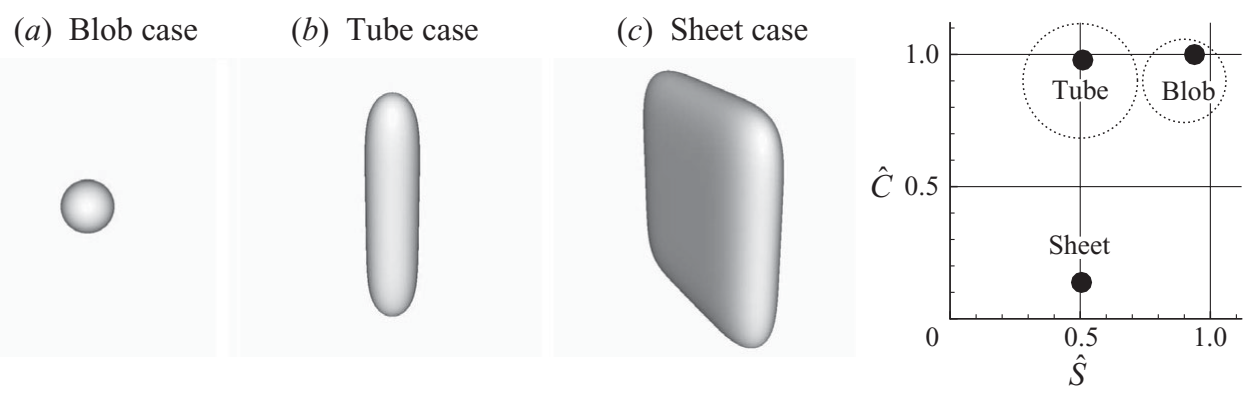

FIGURE 13. Iso-surfaces of three initial fields for the contour level $\phi_{0}^{c}=0.95$ and corresponding feature centres on the $\hat{S}-\hat{C}$ plane with the predominantly blob-, tube- and sheet-like regions sketched.

measures the geometrical global stretching of the individual surface. Taken together, this parameter set defines the geometrical signature of the iso-surface by specifying its location as a point in a multidimensional 'feature-space'.

A subset of the feature space is the so-called visualization space. This comprises a subspace of three parameters $(\hat{S}, \hat{C}, \lambda)$, where feature centres $\hat{S}$ and $\hat{C}$ are respectively dimensionless forms of weighted first-order moments of the joint p.d.f. of $S$ and $C$. Each closed iso-surface is then represented by a single point in the visualization space $(\hat{S}, \hat{C}, \lambda)$. At each scale, the cloud of points in visualization space represents all structures at that scale, for a specified iso-contour value. We remark that, for the full field, iso-surfaces of $\phi$ are topologically invariant in time. This is not the case for the multi-scale component fields after curvelet decomposition, where, in general, many individual disconnected iso-surfaces are generated. Nonetheless, the set of iso-surfaces at each scale can be interpreted as containing information on the statistical geometry of structures at that scale.

Finally, the geometry of a structure is classified by its location $(\hat{S}, \hat{C}, \lambda)$ in the visualization space. An analysis of a generic surface shows that different regions in the visualization space can be viewed as representing different geometry shapes. Bloblike structures occupy the region near the point $(1,1,1)$ that corresponds to spheres. Tube-like structures are localized near the $(1 / 2,1, \lambda)$ axis where $\lambda$ is an indication of how stretched the tube is, and the transition to sheet-like structures occurs as $\hat{C}$ and $\lambda$ decrease. An illustration for three typical geometry shapes in the present study and corresponding feature centres on the $\hat{S}-\hat{C}$ plane with the predominantly blob-, tube- and sheet-like regions sketched are shown in figure 13. More details on the classification and the visualization space $(\hat{S}, \hat{C}, \lambda)$ are shown in figures 6 and 7 of Bermejo-Moreno \& Pullin (2008). The clustering algorithm in the original methodology was not applied in this study.

\subsection{Multi-scale diagnostics}

Next, we analyse the non-local geometry in the evolution of $\phi_{0}^{(1)}$ of $\S 3.3$ corresponding to Lagrangian blobs. Figures 14 and 15 show $x-y$ plane cuts at $z=\pi$ of the full Lagrangian scalar fields with colour intensity proportional to $\phi, 0<\phi \leqslant 1$ for sphere/blob-like initial $\phi_{0}^{(1)}$ at different times $\alpha T_{e}$ in stationary isotropic turbulence and the frozen turbulent field respectively. The evolution of $\phi$ in the two cases is similar in the early stages at $t=T_{e} / 16$ and $t=T_{e} / 8$. Then the Lagrangian structures in turbulence are stretched or wound by dynamic turbulent motions producing alignment between Lagrangian surfaces and vortex lines as discussed in $\$ \S 4.2$ and 4.4. In contrast, 
(a) $t=T_{e} / 16$

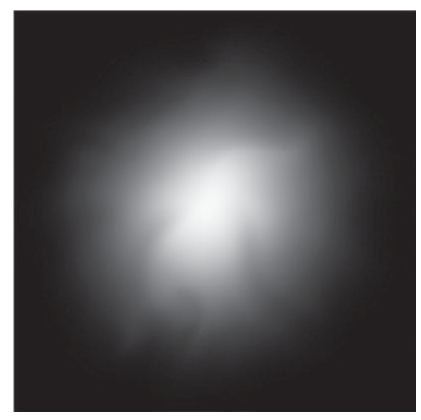

(d) $t=T_{e} / 2$

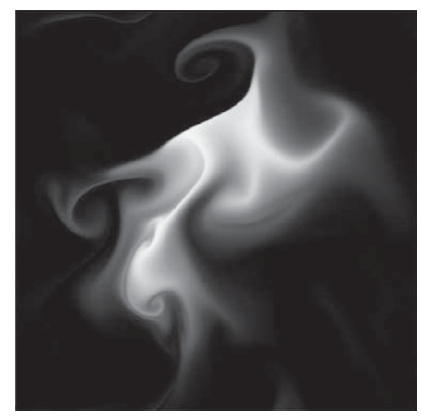

(b) $t=T_{e} / 8$

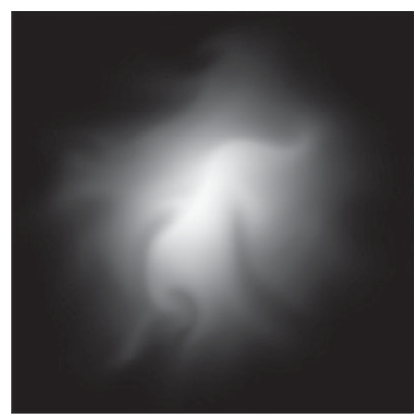

(e) $t=3 T_{e} / 4$

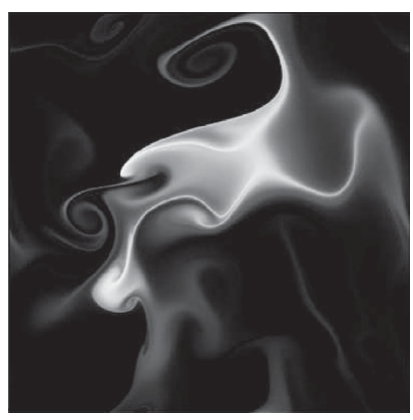

(c) $t=T_{e} / 4$

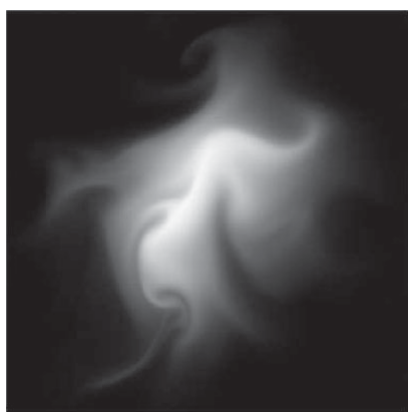

(f) $t=T_{e}$

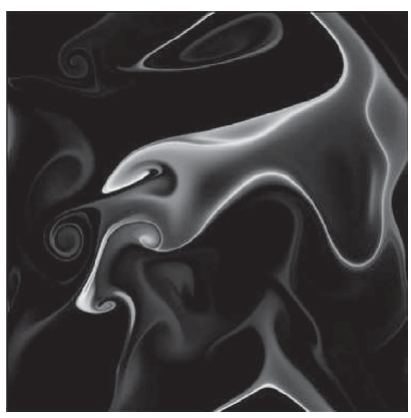

FIGURE 14. Plane cuts normal to the $z$-axis at its midpoint of the original three-dimensional Lagrangian scalar fields with the blob-like initial $\phi_{0}^{(1)}$ in stationary isotropic turbulence at different times.

in the frozen turbulent field, Lagrangian structures are attracted onto a frozen stream surface and are therefore strained only in fixed regions to form more nearly singular local structures as shown in (4.19).

The multi-scale methodology described in $\S 5.1$ was applied to the three-dimensional Lagrangian scalar fields at each instantaneous time. For the given resolution of $1024^{3}$ grid points, we will analyse the first six scales provided by the curvelet transform. They will be denoted by a scale number, from 0 to 5 . Increasing values of the scale number correspond to smaller scales. For example, the plane cuts of $\phi$ at $t=T_{e}$ in turbulence for each one of the filtered scales resulting from the multi-scale analysis are shown in figure 16, which corresponds to the original field shown in figure $14(f)$.

Volume-data p.d.f.s obtained for the Lagrangian scalar field for each one of the filtered scales are shown in figure 17. The widening of the p.d.f.s at small scales indicates that there is an increasing population of small-scale structures appearing in the Lagrangian scalar field with increasing time: this corresponds to the cascade process in Fourier space as illustrated in figure 6. In figure 18, exponential tails in normalized p.d.f.s at intermediate and small scales are observed. This shows the strong intermittency in the Lagrangian scalar field at $t=T_{e}$, which corresponds to the sheet-like small-scale structures as shown in figures $14(f)$ and $16(d-f)$. The spectra of $\phi$ at $t=T_{e}$ associated with the original scalar field for each one of the filtered scales are shown in figure 19. Combined with figure 14, we observe localization in both Fourier and physical domains through the curvelet filtering. 
(a) $t=T_{e} / 16$

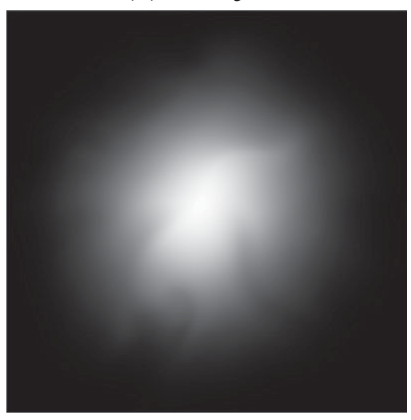

(d) $t=T_{e} / 2$

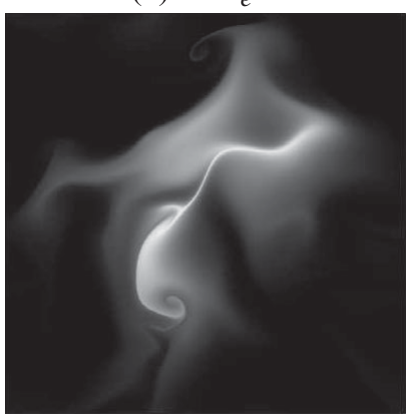

(b) $t=T_{e} / 8$

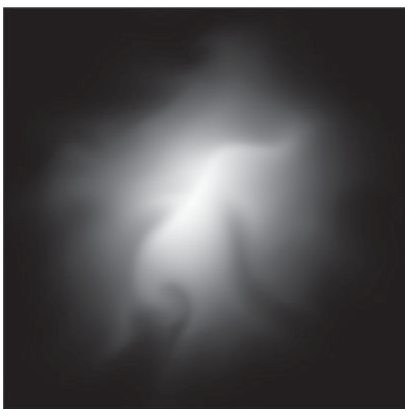

(e) $t=3 T_{e} / 4$

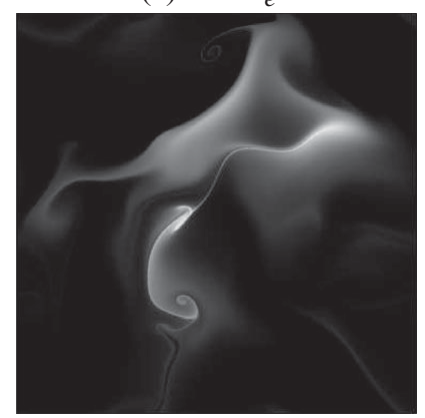

(c) $t=T_{e} / 4$

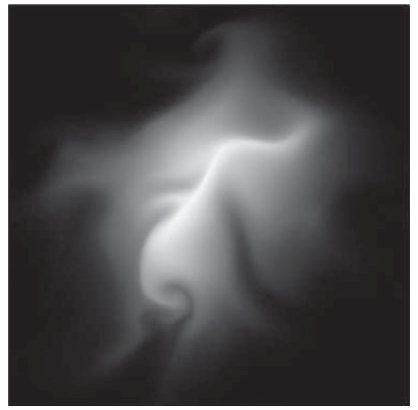

(f) $t=T_{e}$

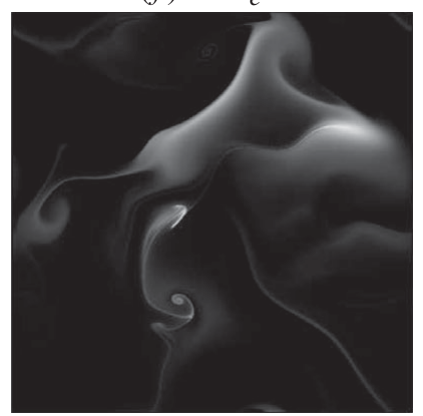

FIGURE 15. Plane cuts normal to the $z$-axis at its midpoint of the original three-dimensional Lagrangian scalar fields with the blob-like initial $\phi_{0}^{(1)}$ in the frozen turbulent field at different times.

\subsection{Geometry of Lagrangian structures}

Iso-surfaces of $\phi\left(\boldsymbol{x}, \alpha T_{e}\right)$ with three different initial conditions of $\S 3.3$ were obtained for each of the filtered scales after the multi-scale decomposition. The contour values $\phi_{i}^{c}$ at scale $i$ are computed from the volume p.d.f.s as

$$
\phi_{i}^{c}=\left\langle\phi_{i}\right\rangle+2 \sqrt{\operatorname{Var}\left(\phi_{i}\right)}+\Delta, \quad i=1 \sim 5,
$$

with $\Delta=\left|\phi_{\max }-\phi_{\min }\right| \times 5 \%$. This choice eliminates artificial, tiny high-frequency oscillations induced by the curvelet transform near the boundary of computational domain. We argue that the sample of iso-surfaces corresponding to the same relative contour value represents the typical statistical geometric properties at each scale. An additional step in the extraction is applied to periodically reconnect those structures that penetrate the box boundaries, to properly account for their geometry.

The largest scale $i=0$ strongly depends on the initial conditions. This is of less relevance in this analysis and the contouring for scale 0 was only applied for the iso-contour level $\phi_{0}^{c}=0.95$ at $t=0$ and $t=T_{e} / 16$ to show the difference among three initial conditions. Corresponding iso-surfaces of three initial fields and feature centres on the $\hat{S}-\hat{C}$ plane are shown in figure 13 . A minimum number of points, $N_{\min }=1500$, is considered a threshold for a structure to be analysed, so that it is sufficiently smooth for a reliable calculation of its differential-geometry properties. We remark that this number is higher than $N_{\min }=300$ used by Bermejo-Moreno \& Pullin (2008) and Bermejo-Moreno et al. (2009), because near-singular structures tend to be more relevant in Lagrangian fields than in Eulerian scalar or enstrophy fields with a finite 
(a) Scale 0

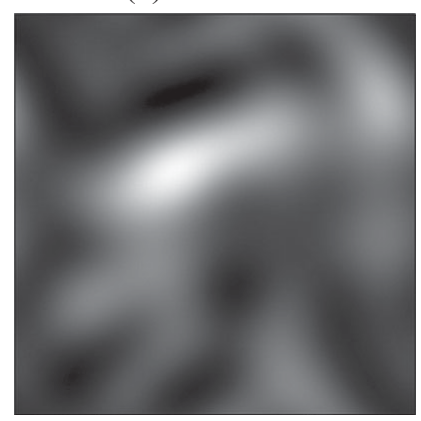

(d) Scale 3

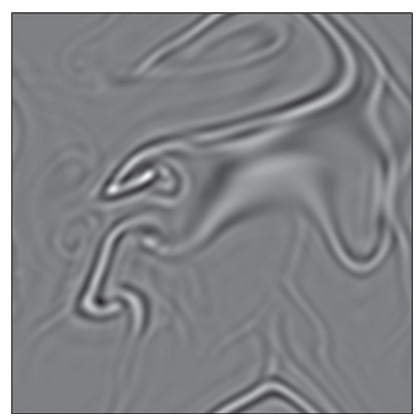

(b) Scale 1

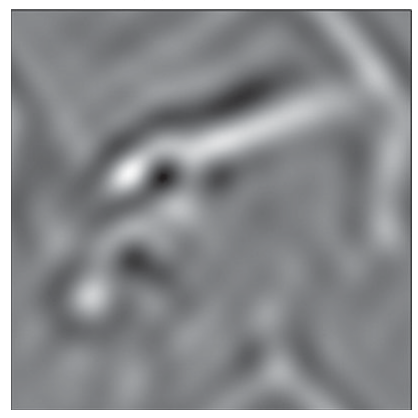

(e) Scale 4

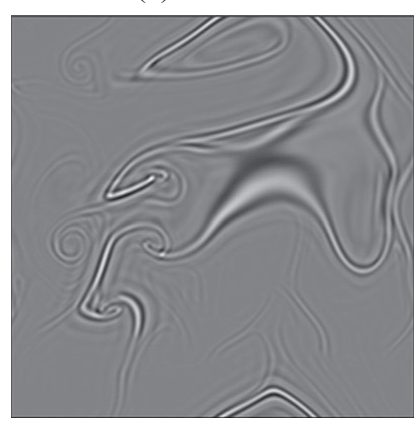

(c) Scale 2

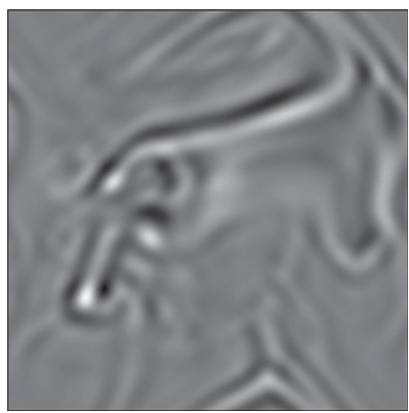

(f) Scale 5

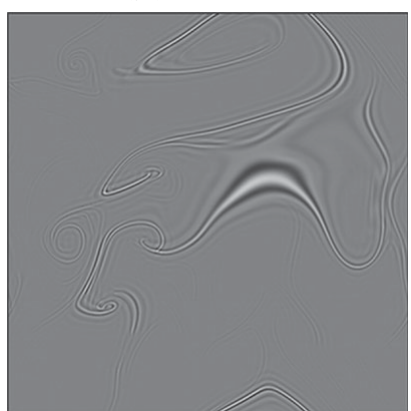

Figure 16. Plane cuts normal to the $z$-axis at its midpoint of the Lagrangian scalar field at $t=T_{e}$ for the blob-like initial $\phi_{0}^{(1)}$ in stationary isotropic turbulence for each one of the filtered scales resulting from the multi-scale analysis.
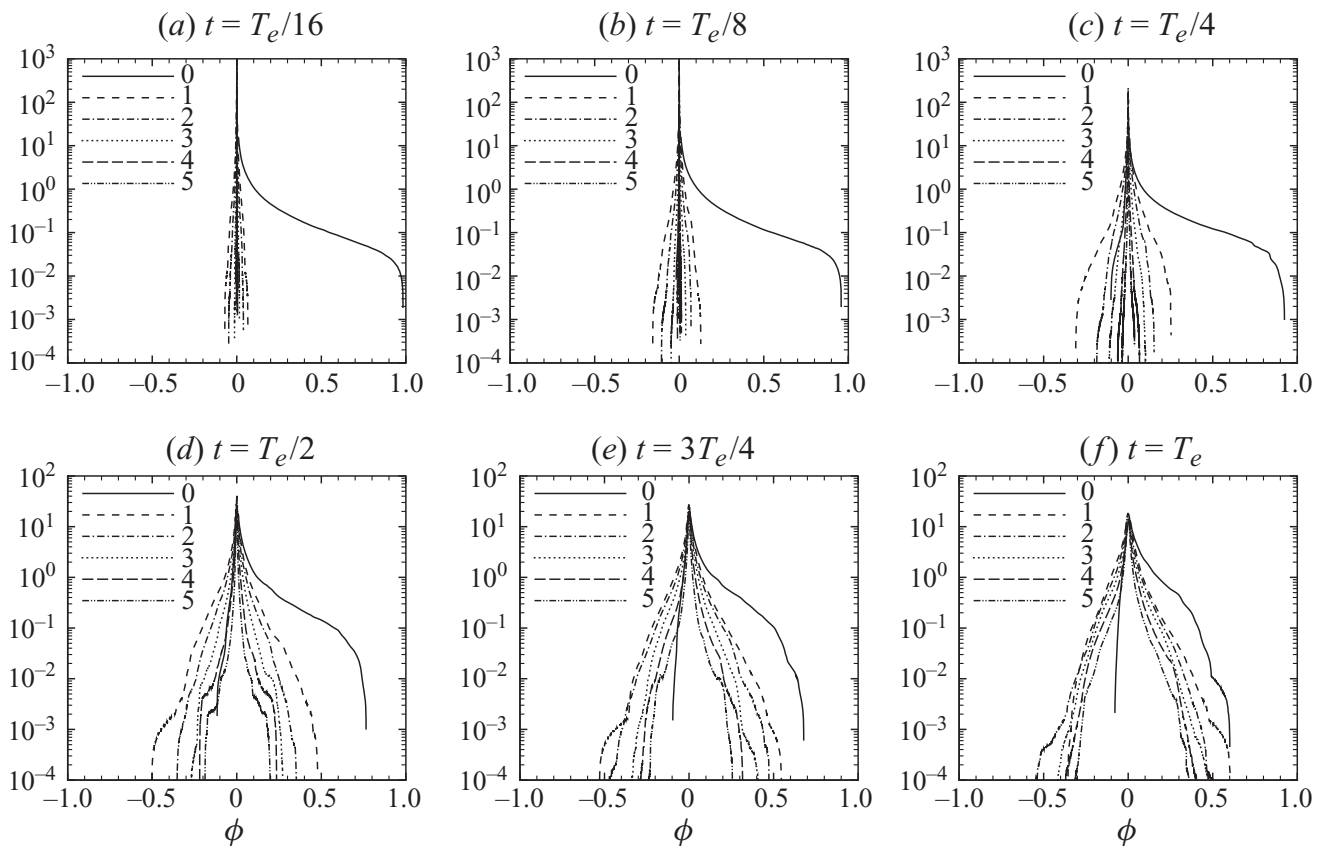

FIGURE 17. Volume-data p.d.f.s of Lagrangian component scalar fields of different scales with $\phi_{0}^{(1)}$ at different times in stationary isotropic turbulence. 


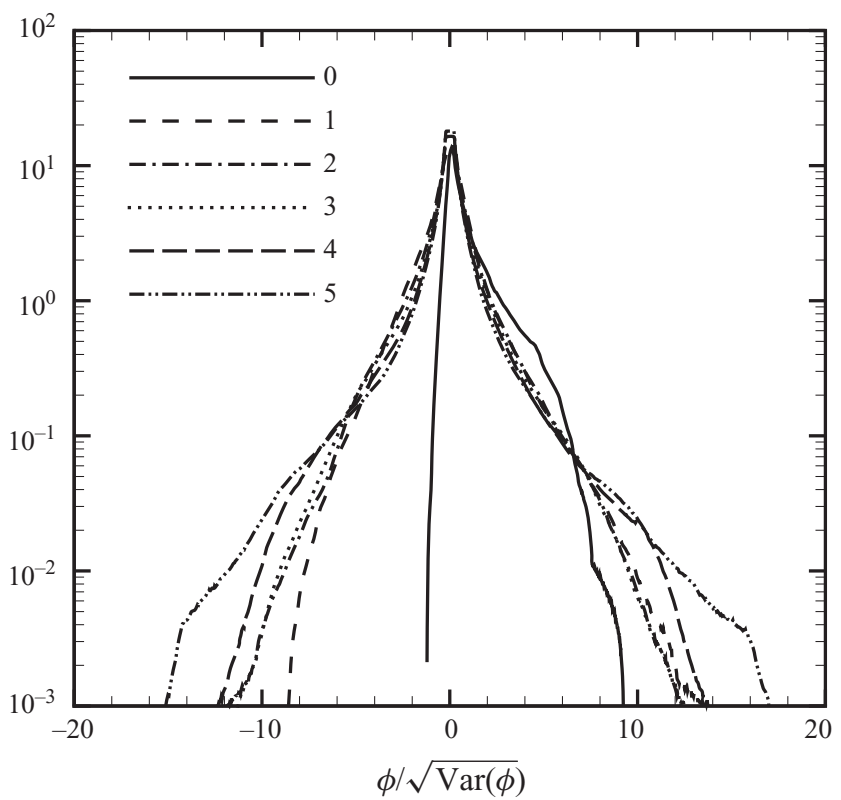

FIGURE 18. Normalized volume-data p.d.f.s of Lagrangian component scalar fields of different scales with $\phi_{0}^{(1)}$ at $t=T_{e}$ in stationary isotropic turbulence.

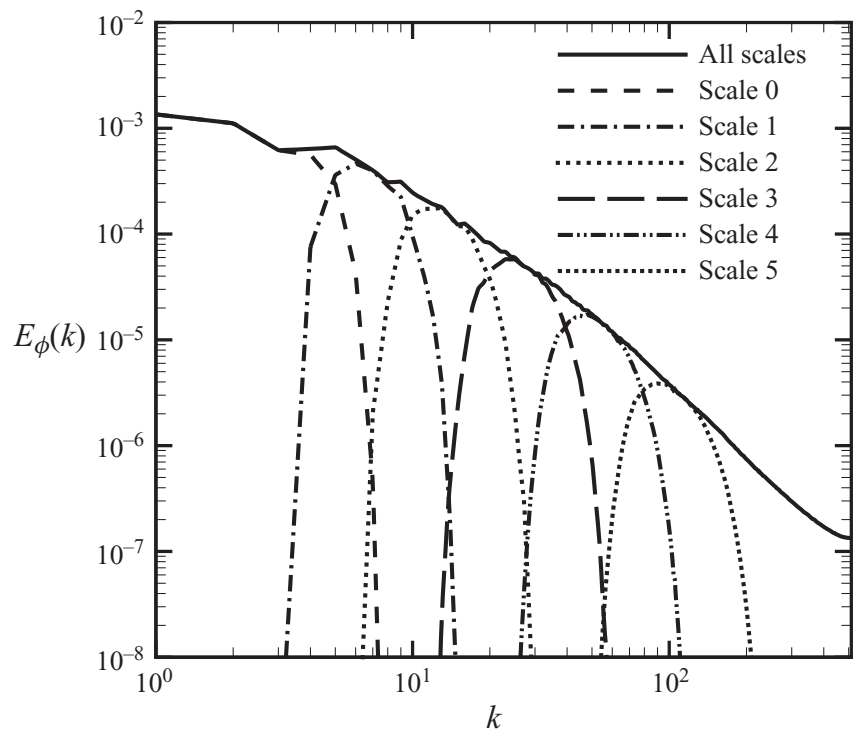

FIGURE 19. Spectra of the full Lagrangian scalar field and component fields with $\phi_{0}^{(1)}$ at $t=T_{e}$.

viscosity. This threshold avoids spurious features from fragmentation while retaining most sample structures in the characterization step for this case.

The structures at each scale in turbulence are now characterized and represented in the visualization space $(\hat{S}, \hat{C}, \lambda)$ as described in $\S 5.1$. Figures 20 and 21 show the depiction of the multi-scale iso-surfaces in visualization space and the $\hat{S}-\hat{C}$ plane, 
(a) $t=T_{e} / 16$

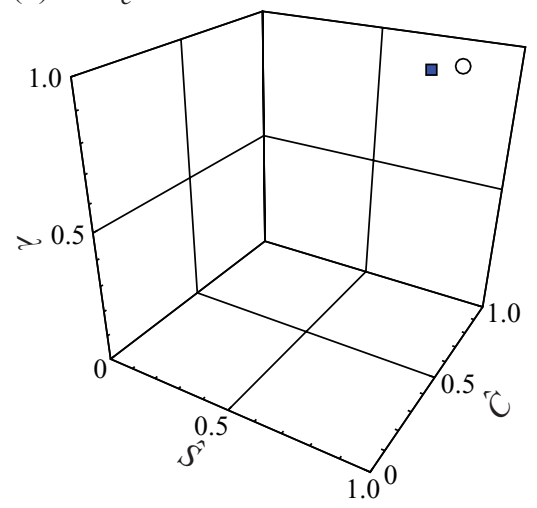

(c) $t=T_{e} / 4$

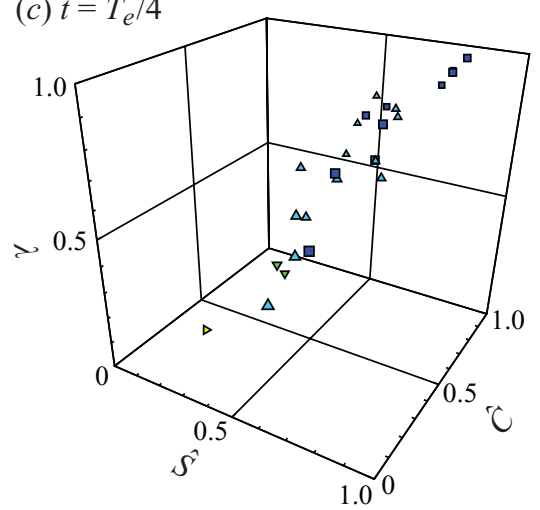

(e) $t=3 T_{e} / 4$

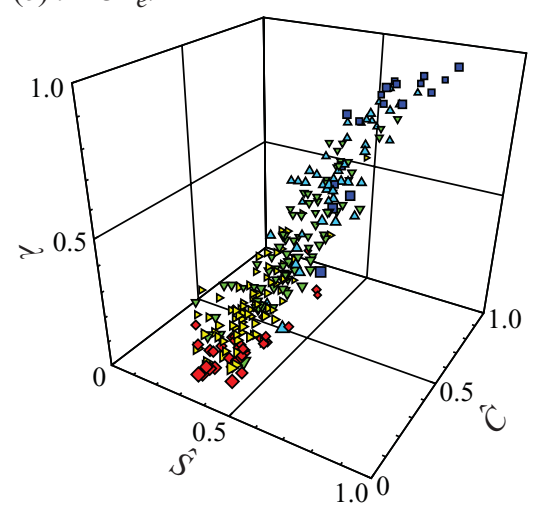

(b) $t=T_{e} / 8$

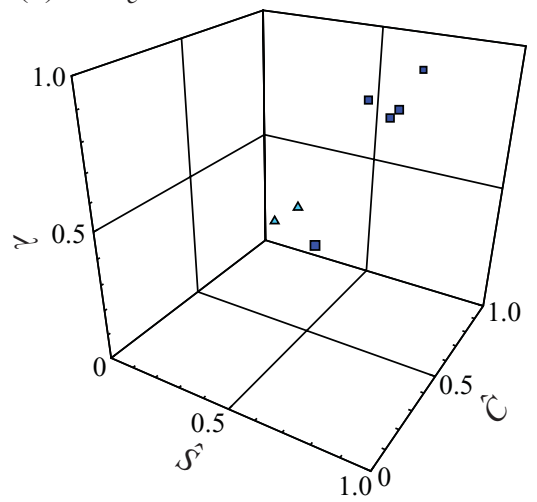

(d) $t=T_{e} / 2$

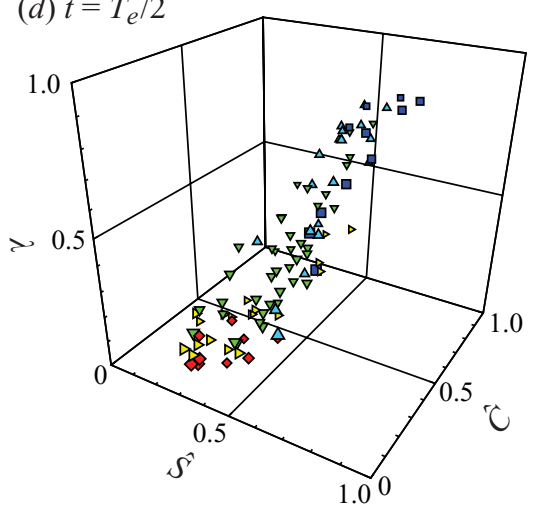

(f) $t=T_{e}$

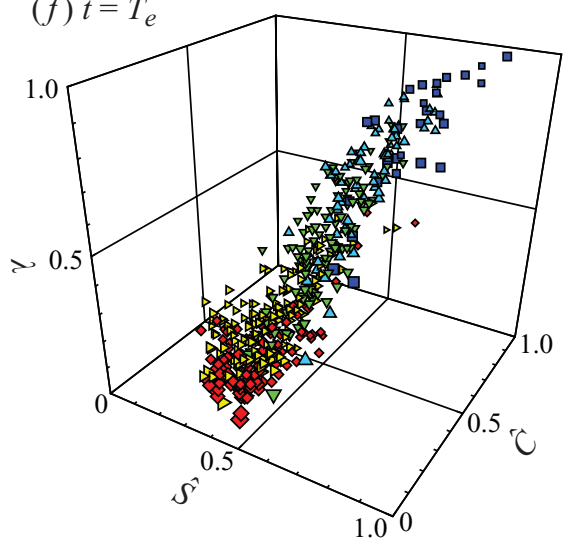

FIGURE 20. (Colour online) Visualization space for Lagrangian structures with the blob-like initial $\phi_{0}^{(1)}$ at different scales in stationary isotropic turbulence ( $\bigcirc$ scale 0 ; $\square$ scale $1 ; \triangle$ scale 2 ; $\nabla$ scale $3 ; \triangleright$ scale 4 ; $\diamond$ scale 5 ). The sizes of symbols are scaled by the log of surface area.

a corresponding subspace, with increasing $t$. Each component graph shows all isosurfaces across all scales colour coded for scale as indicated in the captions. Sizes of symbols in these figures are scaled by the logarithm of the surface area of the structures. At $t=T_{e} / 16$, there are only a few iso-surfaces that are clustered in the 
(a) $t=T_{e} / 16$

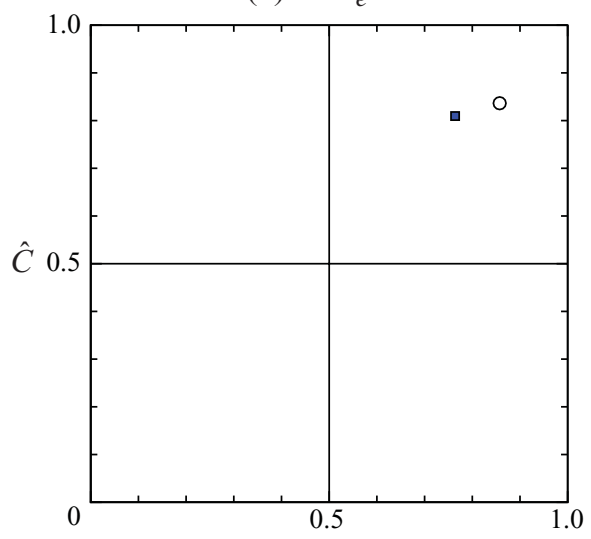

(c) $t=T_{e} / 4$

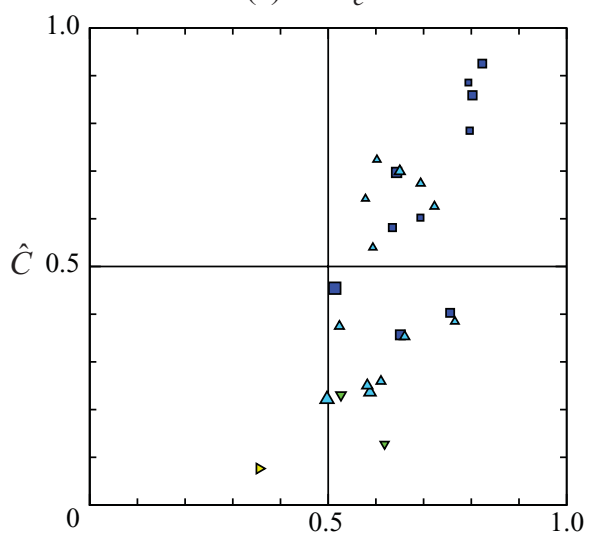

(e) $t=3 T_{e} / 4$

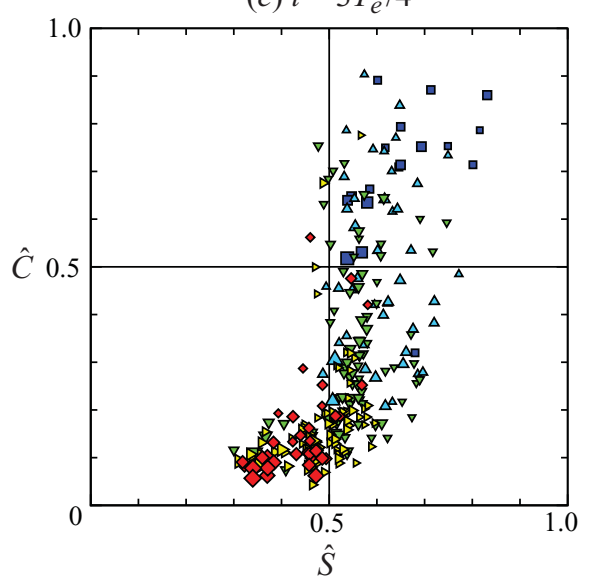

(b) $t=T_{e} / 8$

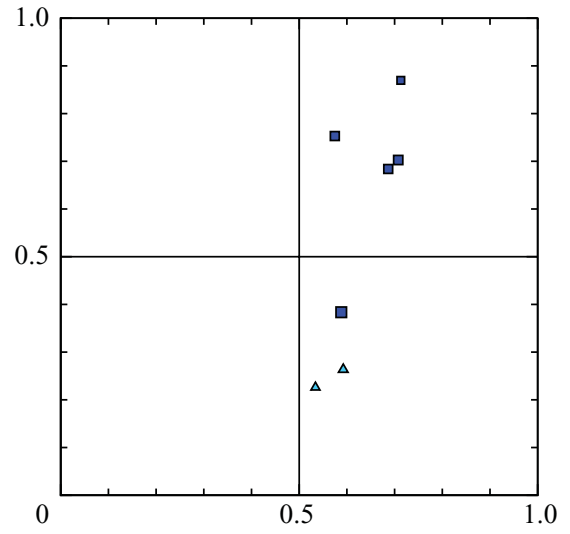

(d) $t=T_{e} / 2$

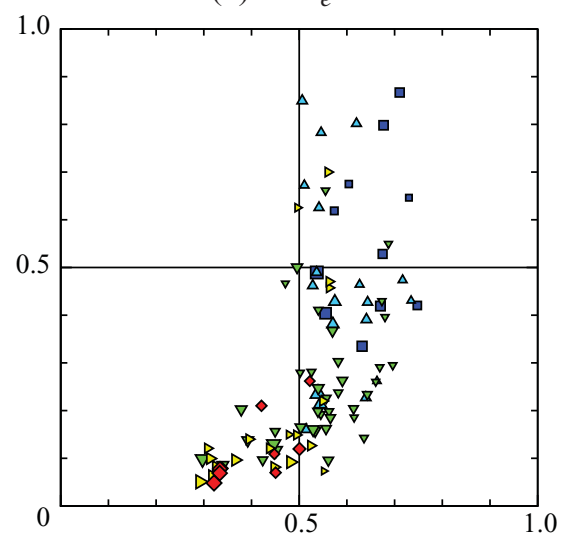

(f) $t=T_{e}$

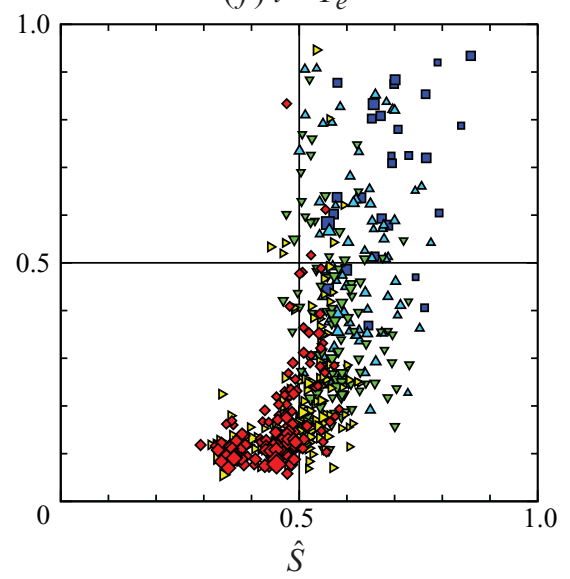

FigurE 21. (Colour online) The $\hat{S}-\hat{C}$ plane of the visualization space for Lagrangian structures with the blob-like initial $\phi_{0}^{(1)}$ at different scales in stationary isotropic turbulence $(\bigcirc$ scale 0 ; $\square$ scale $1 ; \triangle$ scale $2 ; \nabla$ scale 3 ; $\triangleright$ scale 4 ; $\diamond$ scale 5 ). The sizes of symbols are scaled by the $\log$ of surface area. 
upper right of the visualization space in the view shown. This corresponds to bloblike structures. As $t$ increases, progressively, most structures are stretched towards the sheet-like region with low $\hat{C}$ and $\lambda$, while we find only a few structures that appear to migrate first through the tube-like region near the axis $\{\hat{S}=1 / 2, \hat{C}=1, \lambda\}$. For example, at $t=T_{e} / 2$, structures with low values of $\hat{C}$ are dominant, and a few get close to the tube-like region in figure $21(d)$. This could be interpreted as the evolutionary breakdown of Lagrangian blobs that first distort, while a few portions might be rolled up into tube-like structures, and then are either broken down or stretched laterally into sheets or else are perhaps part of vortices whose velocity fields strain small-scale structures into sheet-like form.

Furthermore, it seems the appearance of tube-like structures occurs later in that time evolution. In figure 21(e,f), between $t=3 T_{e} / 4$ and $t=T_{e}$, more structures appear in the vicinity of the tube-like region. Details of the multi-scale decomposition within the visualization space of the component fields at $t=T_{e}$ with the blob-like initial condition $\phi_{0}^{(1)}$ are shown in figure 22. This depicts clouds of points, corresponding to each of scales $1-5$ in the $\hat{S}-\hat{C}$ plane. We observe a geometrical progression from blobs through tubes to sheet-like structures with decreasing length scale. Here, we note that a spiral-like sheet of small thickness could fill a substantial volume with the space in between its turns deformed into a tube-like geometry as the sheet rolls up. After filtering, this composite structure would appear as a virtual tube with a larger cross-sectional scale, in terms of averaged radius, than the thickness of the sheet itself.

It is notable that the region of visualization space occupied by the cloud of all Lagrangian scale structures at $t=T_{e}$ is similar in shape to the region of visualization space occupied by the Eulerian enstrophy and dissipation across all scales in figures 12 and 13 of Bermejo-Moreno et al. (2009). The former is a consequence of Lagrangian evolution whilst the latter are instantaneous Eulerian fields. There are differences, however, in which Lagrangian structures tend to show more sheet-like geometry at intermediate and small scales. The cloud of Lagrangian structures at scale 1 in the visualization space occupies a similar region of the visualization space to that of the Eulerian enstrophy at scale 3 (Bermejo-Moreno et al. 2009), and a passive scalar with $S c=0.7$ at scale 4 (Bermejo-Moreno \& Pullin 2008). The corresponding wavenumber range of scale 1 is approximately between 3 and 20. This implies that the geometry transition of Lagrangian structures from blob-like shape to sheet-like shape begins earlier in scale space than for Eulerian fields. This is possibly due to the lack of viscous dissipation for Lagrangian evolution. As described in $\S 4.4$, Lagrangian structures are exposed by persistent straining motions and wound by vortices, but are not subject to smoothing by a dissipation mechanism. The dominant sheet-like structures with strong scalar gradients have an impact on accelerating passive scalar variance dissipation with a finite diffusivity and cause strong intermittency in passive scalar statistics (see Warhaft 2000). Furthermore, the similar-shaped clouds at scales 4 and 5 in figure 22 might imply the self-similar geometry of Lagrangian structures at small scales, which could provide some support for structure-based subgrid-scale modelling. To better investigate this possible self-similar geometry feature, simulations of $\phi$ in higher Reynolds number turbulence with a larger inertial range could be helpful. This requires, however, much higher resolution for both the turbulent field and the Lagrangian scalar field than is employed presently.

Results from the multi-scale geometric analysis applied to Lagrangian structures in the frozen turbulent field are shown in figures 22 and 23. We find a similar geometry transition as that in turbulence but with less small-scale structures at the later stages, which is consistent with the comparison of spectra shown in figure 6 . 
(a) Scale 1
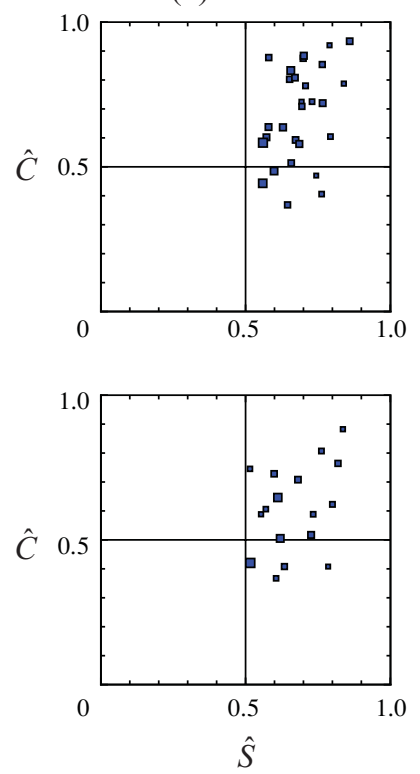

(d) Scale 4
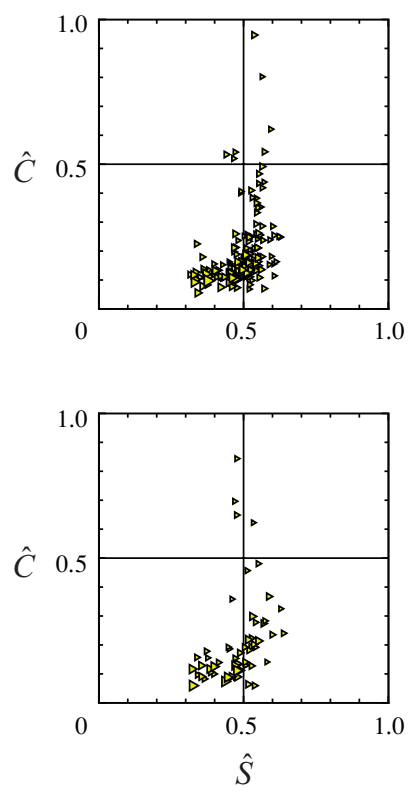

(b) Scale 2
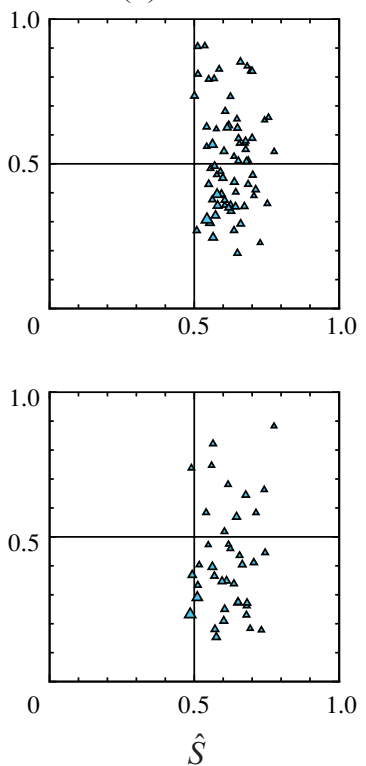

(e) Scale 5
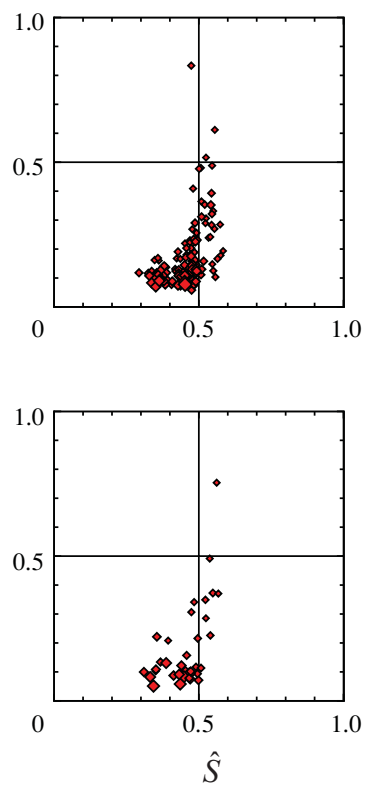

(c) Scale 3
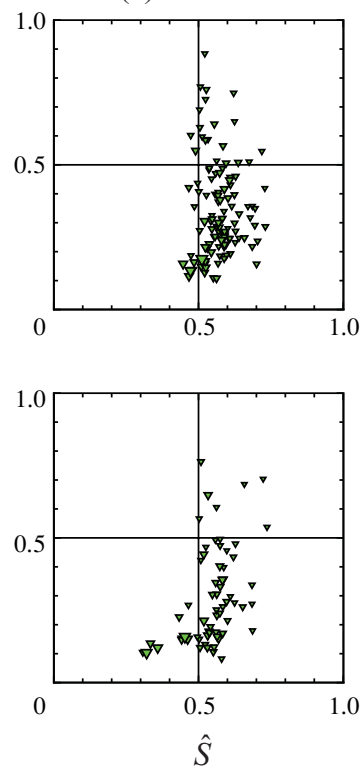

Figure 22. (Colour online) The $\hat{S}-\hat{C}$ plane of the visualization space for each one of the filtered scales with the blob-like initial field $\phi_{0}^{(1)}$ at $t=T_{e}$ in stationary isotropic turbulence (upper row in each subfigure) and the frozen turbulent field (lower row in each subfigure). The sizes of symbols are scaled by the log of surface area.

Moreover, in general, smaller $\hat{S}$ and $\hat{C}$ at intermediate and small scales indicate that Lagrangian structures in the frozen velocity field exhibit more sheet-like shapes. These observations can be better understood by considering average feature centres over all the structures obtained from the extraction step in the multi-scale decomposition 
(a) $t=T_{e} / 2$

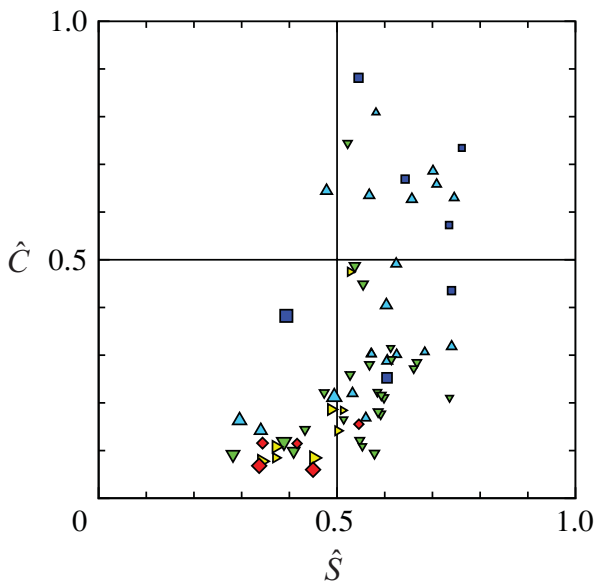

(b) $t=T_{e}$

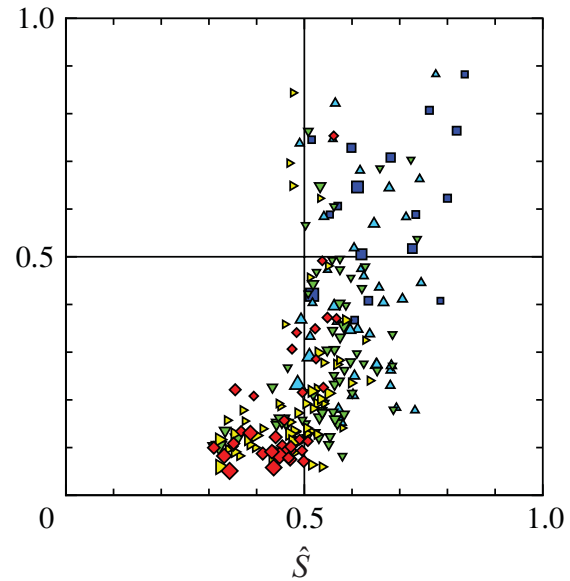

FIgURE 23. (Colour online) The $\hat{S}-\hat{C}$ plane of the visualization space for Lagrangian structures with the blob-like initial $\phi_{0}^{(1)}$ at different scales in the frozen turbulent field ( $\square$ scale $1 ; \triangle$ scale $2 ; \nabla$ scale $3 ; \triangleright$ scale $4 ; \diamond$ scale 5 ). The sizes of symbols are scaled by the log of surface area.

for each scale. The three average geometric feature centres in visualization space are plotted in figure 24. The differences of $\langle\hat{C}\rangle_{i},\langle\hat{S}\rangle_{i}$ and $\langle\lambda\rangle_{i}$ in the two flow cases show that near-singular structures tend to be formed more easily in the frozen turbulent field, because strong straining regions are always located at the same spatial regions as clearly indicated in figures 3 and 15 .

The evolution of Lagrangian structures with the tube-like and sheet-like initial $\phi$-fields corresponding to $\phi_{0}^{(2)}$ and $\phi_{0}^{(3)}$ of $\S 3.3$ in turbulence is shown in figures 25 and 26 , respectively. At early times, the geometries of large-scale structures represented by the feature centres at scale 0 at $t=T_{e} / 16$ are different in all three cases. But we observe that all fields evolve so as to produce similar-shaped clouds of structures at $t=T_{e}$, which is verified by similar average feature centres at each scale shown in figure 24. This suggests that the cloud shape shown in figure $21(f)$ is a Lagrangian attractor that is sensibly independent of the details of initial $\phi$. In this interpretation, the memory of the initial geometric property of the Lagrangian fades after the Lagrangian integral time $T_{L} \approx 3 T_{e} / 4$ in chaotic motion, where $T_{L}$ is obtained from the Lagrangian velocity autocorrelation (Yang et al. 2008). An alternative but perhaps related explanation is that the similarity, in visualization space at late times, of all three present evolved Lagrangian fields and instantaneous Eulerian fields could be a consequence of the statistically steady character of the underlying forced box turbulence DNS, i.e. Lagrangian structures tend to follow and to be attracted to vortex surfaces in high-Reynolds-number turbulence.

\section{Conclusions}

In this study, the particle backward-tracking method was applied to the computation of the temporal evolution of high-resolution Lagrangian scalar fields $\phi(x, t)$ in velocity fields from unsteady, forced, stationary isotropic turbulence and also from a velocity field obtained from a turbulence simulation but frozen in time. Lagrangian structures were extracted as iso-surfaces of the Lagrangian scalar field $\phi(x, t)$. From the evolution of finite-sized Lagrangian structures in turbulence, exponential surface area growth 

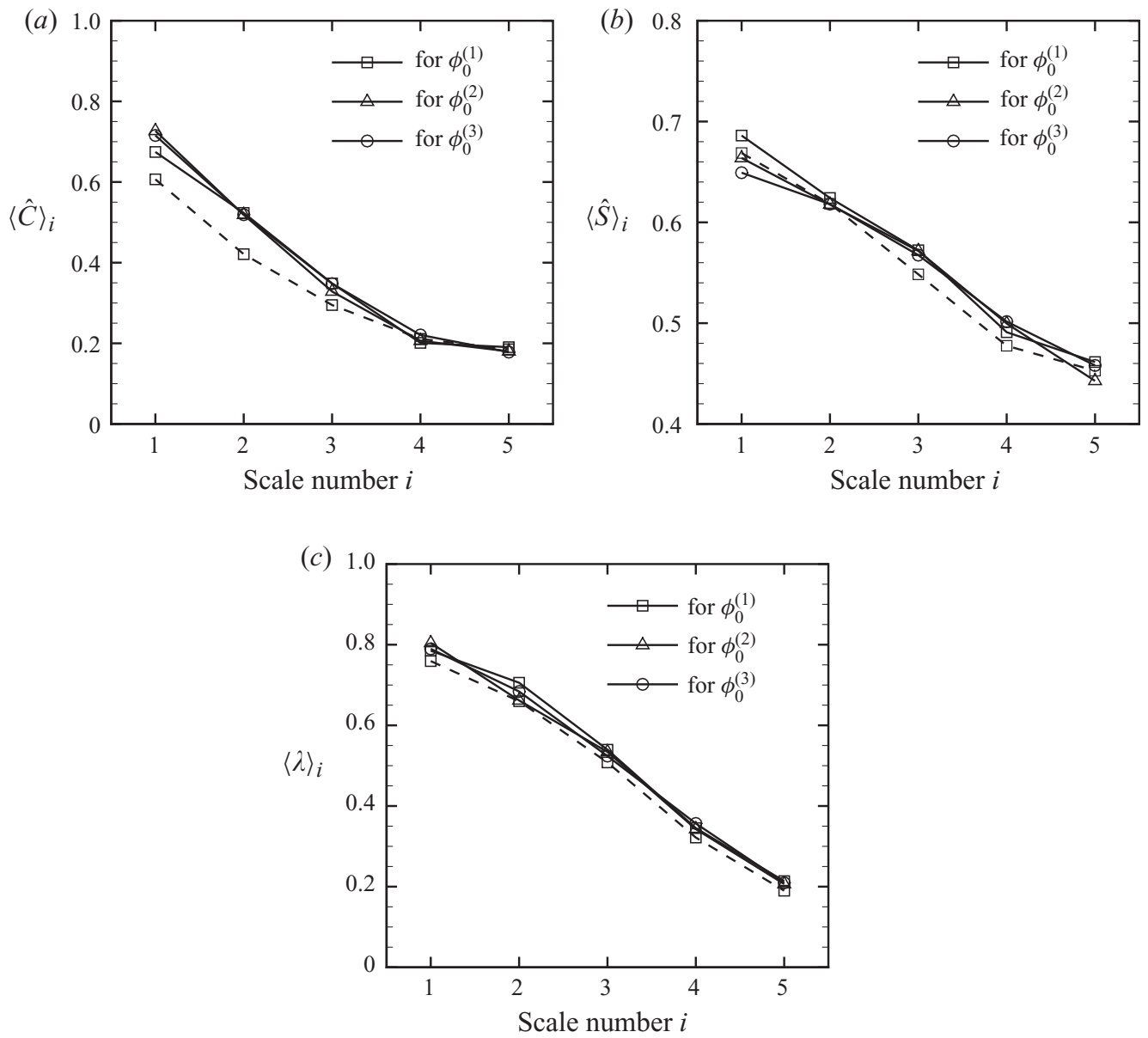

FIgURE 24. Average feature centres at different scales for the Lagrangian scalar field with different initial conditions at $t=T_{e}$ in stationary isotropic turbulence (solid lines) and the frozen turbulent field (dashed lines).

is verified and the growth rate normalized by the reciprocal of the Kolmogorov time scale is approximately $0.33 \pm 0.4$. Stretched structures are observed in highly straining flow regions and rolled-up structures are found in stretched vortex tubes. The formation of rolled-up or spiral structures in the Lagrangian scalar field is consistent with observed alignment between the scalar gradient and vorticity, and the moderate normal alignment scalar gradient and the local velocity. A simple two-dimensional model of a scalar field wound by an axisymmetric vortex reproduces these features.

We find that iso-surfaces of the Lagrangian scalar field are attracted to steady stream surfaces in the frozen velocity field. In contrast, these tend to follow and almost attach to vortex surfaces for the turbulent velocity field, but ultimate, longtime alignment is repressed by the cumulative action of viscous effects. Furthermore, the tendency for the formation of near-singular, sheet-like Lagrangian structures is more apparent in the frozen versus the turbulent velocity field owing to the presence of time-invariant straining regions in the former. It is expected that these two differing alignment scenarios would lead to quite different long-time behaviours and that this constitutes a principal distinction separating the kinematics from the turbulent 
(a) $t=T_{e} / 16$

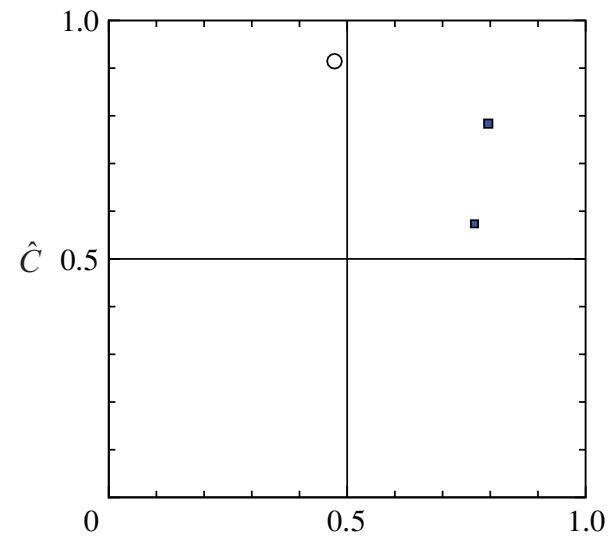

(c) $t=T_{e} / 2$

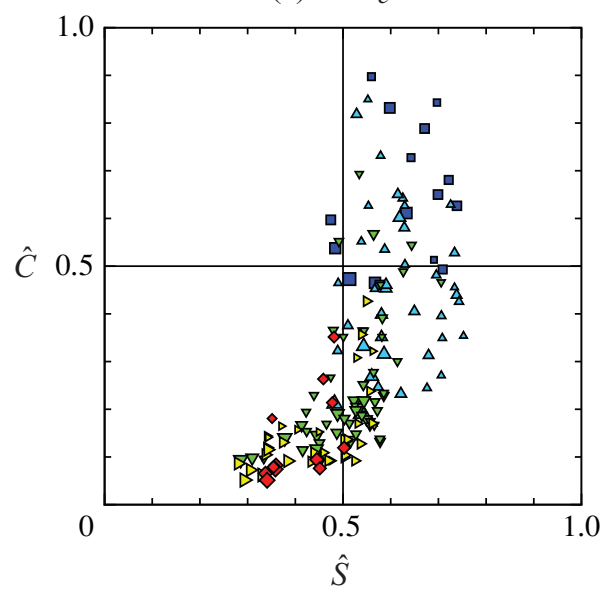

(b) $t=T_{e} / 4$

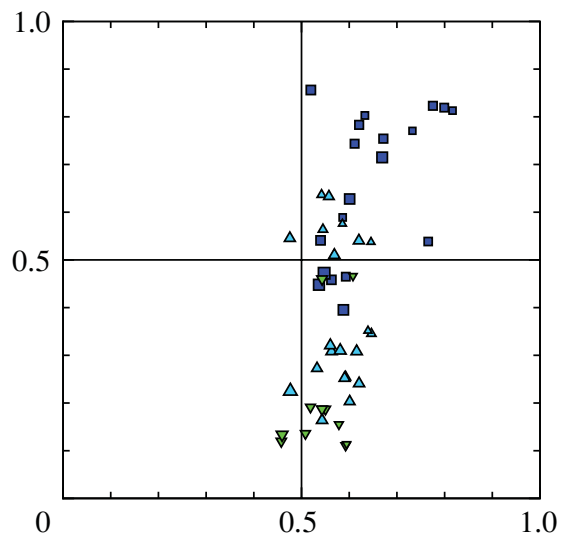

(d) $t=T_{e}$

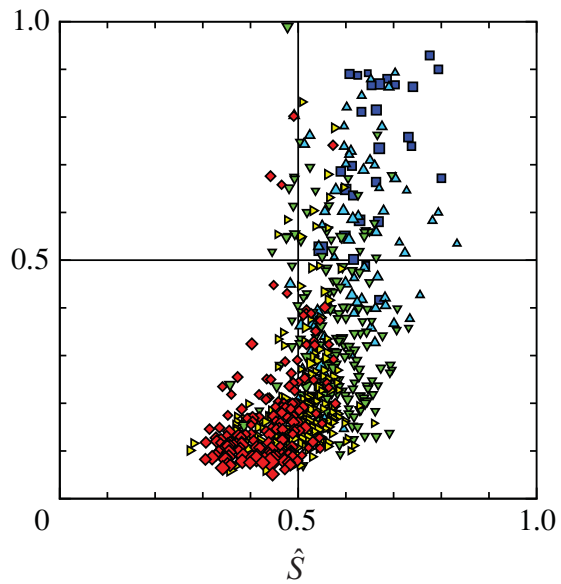

FIGURE 25. (Colour online) The $\hat{S}-\hat{C}$ plane of the visualization space for Lagrangian structures with the tube-like initial $\phi_{0}^{(2)}$ at different scales in stationary isotropic turbulence $(\bigcirc$ scale 0 ; $\square$ scale $1 ; \triangle$ scale $2 ; \nabla$ scale 3 ; $\triangleright$ scale 4 ; $\diamond$ scale 5 ). The sizes of symbols are scaled by the $\log$ of surface area.

dynamics of Lagrangian structures. Moreover, we suggest that this could lend support to hypotheses both concerning geometric similarities between Lagrangian scalar fields and corresponding vorticity fields, and also the independence of geometric signatures of Lagrangian structures on initial conditions of the Lagrangian scalar field.

A multi-scale geometric analysis (Bermejo-Moreno \& Pullin 2008) was used to study the statistical, evolutionary geometry of the Lagrangian scalar field for both the turbulent and the frozen velocity fields. The evolutionary breakdown of Lagrangian blobs, from $t=0$ to $t=T_{e}$, was represented in a space of reduced geometrical parameters representative of the shape of individual structures. As time increases, we observed that, for the turbulent velocity field, multi-scale structures appear to migrate from the blob-like region to the sheet-like region of that space. A few largescale, tube-like structures appear at later times because of the roll-up phenomenon. Somewhat similar behaviour was qualitatively observed for the frozen field, but the population of small-scale structures at $t=T_{e}$ was found to be much diminished in comparison with the turbulent velocity field. In comparison with instantaneous passive 
(a) $t=T_{e} / 16$

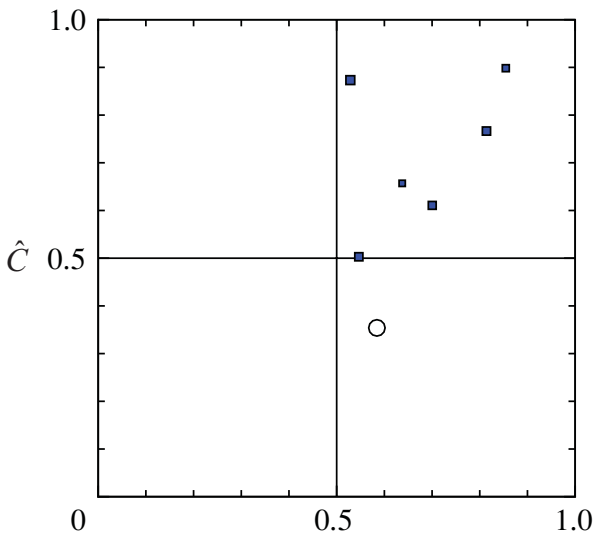

(c) $t=T_{e} / 2$

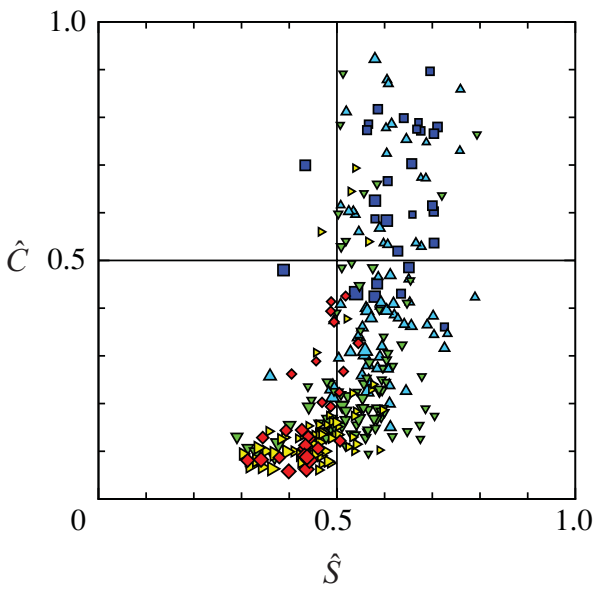

(b) $t=T_{e} / 4$

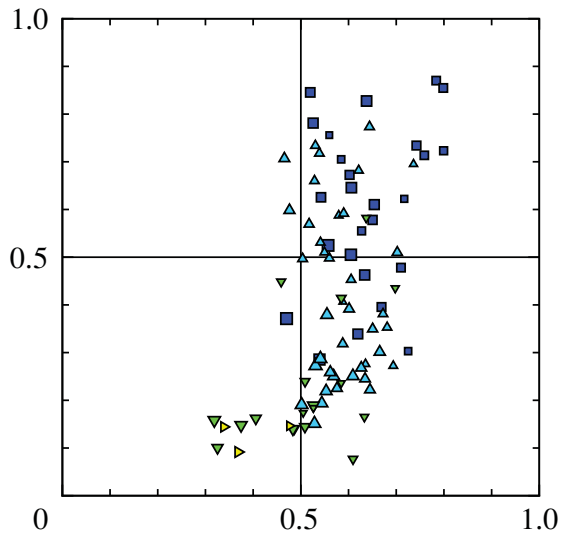

(d) $t=T_{e}$

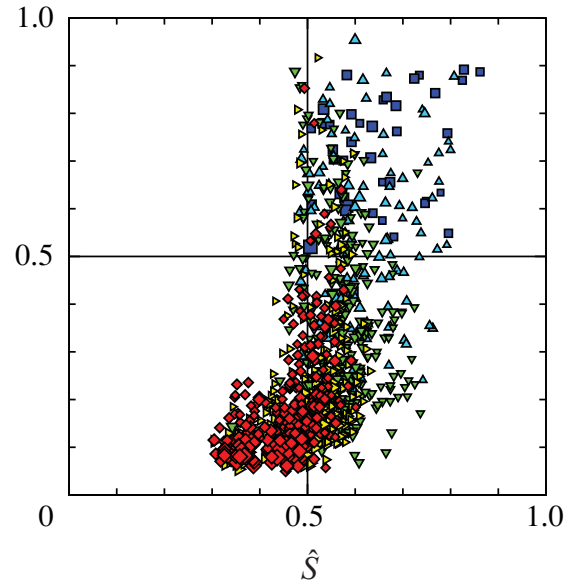

FIGURE 26. (Colour online) The $\hat{S}-\hat{C}$ plane of the visualization space for Lagrangian structures with the sheet-like initial $\phi_{0}^{(3)}$ at different scales in stationary isotropic turbulence $(\bigcirc$ scale 0 ; $\square$ scale $1 ; \triangle$ scale $2 ; \nabla$ scale $3 ; \triangleright$ scale $4 ; \diamond$ scale 5 ). The sizes of symbols are scaled by the $\log$ of surface area.

scalar and enstrophy fields in turbulence studied by Bermejo-Moreno \& Pullin (2008) and Bermejo-Moreno et al. (2009), Lagrangian structures show predominantly more sheet-like geometry in intermediate and small scales, perhaps owing to the lack of viscous dissipation. Moreover, the geometry of Lagrangian structure from blob-like, tube-like and sheet-like initial $\phi$-fields is very similar at $t=T_{e}$. This could imply that the geometry of Lagrangian structures at the later stage does not depend on initial configurations.

A natural line of extension of this work is to the study on the geometry of Lagrangian structures in a turbulent wall-bounded flow. This could help clarify the mechanism of turbulent coherent near-wall structures that are still not well understood, and which is important for drag reduction, turbulent control and other possible applications (Robinson 1991). Recent developments on curvelets (see Demanet \& Ying 2007) allow their extension to non-periodic domains by means of the mirror-extended curvelets with no penalty in the redundancy or computational complexity with respect to their periodic counterparts, and can be easily included. 
Furthermore, the methodology for the geometric analysis is applicable to experimental data sets. The multi-scale decomposition in the extraction step is based on the curvelet transform and is currently implemented to treat domain boundaries by periodization. The characterization and classification of educed structures (Bermejo-Moreno \& Pullin 2008) can then be immediately applied.

Another possible direction is the investigation of the evolution of vortex surfaces. In an inviscid flow, vortex surfaces are Lagrangian structures, but little is understood concerning their evolutionary geometry. An interesting choice of the initial Lagrangian scalar field is to choose iso-surfaces of $\phi_{0}$ to be vortex surfaces in some particular initial flow fields, e.g. Taylor-Green (Brachet et al. 1983) or Kida-Pelz initial velocity fields (Boratav \& Pelz 1994). Further research on this issue could shed light on basic Lagrangian mechanisms of turbulence and vortex dynamics.

This work has been supported in part by the National Science Foundation under grant DMS-0714050. D. I. Pullin benefited from support during a visit to the Center for Water Research at the University of Western Australia.

\section{REFERENCES}

Aref, H. 1984 Stirring by chaotic advection. J. Fluid Mech. 143, 1-21.

Ashurst, W. T., Kerstein, A. R., Kerr, R. M. \& Gibson, C. H. 1987 Alignment of vorticity and scalar gradient with strain rate in simulated Navier-Stokes turbulence. Phys. Fluids 30, $2343-2353$.

BAtchelor, G. K. $1952 a$ Diffusion in a field of homogeneous turbulence. Part II. The relative motion of particles. Proc. Cambridge Phil. Soc. 48, 345-362.

BATCHELOR, G. K. $1952 b$ The effect of homogeneous turbulence on material lines and surfaces. Proc. R. Soc. Lond. A 213, 349-366.

Bermejo-Moreno, I. \& Pullin, D. I. 2008 On the non-local geometry of turbulence. J. Fluid Mech. 603, 101-135.

Bermejo-Moreno, I., Pullin, D. I. \& Horiuti, K. 2009 Geometry of enstrophy and dissipation, grid resolution effects and proximity issues in turbulence. J. Fluid Mech. 620, 121-166.

Boratav, O. N. \& Pelz, R. B. 1994 Direct numerical simulation of transition to turbulence from a high-symmetry initial condition. Phys. Fluids 6, 2757-2784.

Bourgoin, M., Ouellette, N. T., Xu, H. T., Berg, J. \& Bodenschatz, E. 2006 The role of pair dispersion in turbulent flow. Science 311, 835-838.

Brachet, M. E., Meiron, D. I., Orszag, S. A., Nickel, B. G., Morf, R. H. \& Frisch, U. 1983 Small-scale structure of the Taylor-Green vortex. J. Fluid Mech. 130, 411-452.

Brethouwer, G., Hunt, J. C. R. \& Nieuwstadt, F. T. M. 2003 Micro-structure and Lagrangian statistics of the scalar field with a mean gradient in isotropic turbulence. J. Fluid Mech. 474, $193-225$.

Candès, E., Demanet, L., Donoho, D. \& Ying, L. 2005 Fast discrete curvelet transforms. Multiscale Model Simul. 5, 861-899.

Chertkov, M., Pumir, A. \& Shraiman, B. I. 1999 Lagrangian tetrad dynamics and the phenomenology of turbulence. Phys. Fluids 11, 2394-2410.

Chong, M. S., Perry, A. E. \& CAntwell, B. J. 1990 A general classification of three-dimensional flow fields. Phys. Fluids A 2, 765-777.

Chung, D. \& Pullin, D. I. 2009 Large-eddy simulation and wall modelling of turbulent channel flow. J. Fluid Mech. 631, 281-309.

Demanet, L. \& Ying, L. 2007 Curvelets and wave atoms for mirror-extended images. In Society of Photo-Optical Instrumentation Engineers (SPIE) Conference Series, vol. 6701, p. 67010J. SPIE.

FARGE, M. 1992 Wavelet transforms and their applications to turbulence. Annu. Rev. Fluid Mech. 24, 395-457. 
Girimaji, S. S. \& Pope, S. B. 1990 Material-element deformation in isotropic turbulence. J. Fluid Mech. 220, 427-458.

Goto, S. \& KidA, S. 2007 Reynolds-number dependence of line and surface stretching in turbulence: folding effects. J. Fluid Mech. 586, 59-81.

HALler, G. 2001 Distinguished material surfaces and coherent structures in three-dimensional fluid flows. Physica D 149, 248-277.

Hou, T. Y. \& LI, R. 2006 Computing nearly singular solutions using pseudo-spectral methods. J. Comput. Phys. 226, 379-397.

Kraichnan, R. H. 1965 Lagrangian-history closure approximation for turbulence. Phys. Fluids 8, 575-598.

LEONARD, A. 2009 The universal structure of high-curvature regions of material lines in chaotic flows. J. Fluid Mech. 622, 167-175.

LeVeque, R. J. 1992 Numerical Methods for Conservation Laws, 2nd edn. Birkhäuser.

Li, Y. \& Meneveau, C. 2007 Material deformation in a restricted Euler model for turbulent flows: analytic solution and numerical tests. Phys. Fluids 19, 015104.

Lundgren, T. S. 1982 Strained spiral vortex model for turbulent fine structure. Phys. Fluids 25, 2193-2203.

Ma, J., Hussaini, M. Y., Vasilyev, O. V. \& Le Dimet, F.-X. 2009 Multiscale geometric analysis of turbulence by curvelets. Phys. Fluids 21, 075104.

Marsden, J. E. \& Hughes, T. J. R. 1994 Mathematical Foundations of Elasticity. Dover.

Meneveau, C. 1991 Analysis of turbulence in the orthonormal wavelet representation. J. Fluid Mech. 232, 469-520.

Misra, A. \& Pullin, D. I. 1997 A vortex-based subgrid stress model for large-eddy simulation. Phys. Fluids 9, 2443-2454.

Moisy, F. \& JimÉNeZ, J. 2004 Geometry and clustering of intense structures in isotropic turbulence. J. Fluid Mech. 513, 111-133.

Monin, A. S. \& Yaglom, A. M. 1975 Statistical Fluid Mechanics of Turbulence. MIT.

Nahum, A. \& Seifert, A. 2006 Technique for backward particle tracking in a flow field. Phys. Rev. E 74, 016701.

PENG, J. \& DABIRI, J. O. 2008 An overview of a Lagrangian method for analysis of animal wake dynamics. J. Exp. Biol. 211, 280-287.

Pope, S. B. 1987 Turbulent premixed flames. Annu. Rev. Fluid Mech. 19, 237-270.

Pope, S. B., Yueng, P. K. \& Girimaji, S. S. 1989 The curvature of material surfaces in isotropic turbulence. Phys. Fluids A 1, 2010-2018.

Pullin, D. I. \& SAfFMan, P. G. 1993 On the Lundgren-Townsend model of turbulent fine scales. Phys. Fluid A 5, 126-145.

Pumir, A., Shraiman, B. I. \& Chertkov, M. 2000 Geometry of Lagrangian dispersion in turbulence. Phys. Rev. Lett. 85, 5324-5327.

Rhines, P. B. \& Young, W. R. 1983 How rapidly is a passive scalar mixed within closed streamlines? J. Fluid Mech. 133, 133-145.

Robinson, S. K. 1991 Coherent motions in the turbulent boundary layer. Annu. Rev. Fluid Mech. 23, 601-639.

Ruetsch, G. R. \& MAXEY, M. R. 1992 The evolution of small-scale structures in homogeneous isotropic turbulence. Phys. Fluid A 4, 2747-2760.

Salazar, J. P. L. C. \& Collins, L. R. 2009 Two-particle dispersion in isotropic turbulent flows. Annu. Rev. Fluid Mech. 41, 405-432.

SAWford, B. 2001 Turbulent relative dispersion. Annu. Rev. Fluid Mech. 33, 289-317.

Schumacher, J., Sreenivasan, K. R. \& Yeung, P. K. 2005 Very fine structures in scalar mixing. J. Fluid Mech. 531, 113-122.

Stam, J. 1999 Stable fluids. In SIGGRAPH'99: Proceedings of the 26th Annual Conference on Computer Graphics and Interactive Techniques, pp. 121-128. ACM.

TAYlor, G. I. 1922 Diffusion by continuous movements. Proc. Lond. Math. Soc. 20, 196-212.

Toschi, F. \& Bodenschatz, E. 2009 Lagrangian properties of particles in turbulence. Annu. Rev. Fluid Mech. 41, 375-404.

Wang, L. P. \& Peters, N. 2006 The length-scale distribution function of the distance between extremal points in passive scalar turbulence. J. Fluid Mech. 554, 457-475. 
Warhaft, Z. 2000 Passive scalars in turbulent flows. Annu. Rev. Fluid Mech. 32, 203-240.

Xu, H., Ouellette, N. T. \& Bodenschatz, E. 2008 Evolution of geometric structures in intense turbulence. New J. Phys. 10, 013012.

YANG, Y., HE, G.-W. \& WANG, L.-P. 2008 Effects of subgrid-scale modelling on Lagrangian statistics in large-eddy simulation. J. Turbul. 9 (8), 1-24.

YeunG, P. K. 2002 Lagrangian investigations of turbulence. Annu. Rev. Fluid Mech. 34, 115-142.

Yeung, P. K. \& Pope, S. B. 1988 An algorithm for tracking fluid particles in numerical simulations of homogeneous turbulence. J. Comput. Phys. 79, 373-416. 